\title{
Wave Propagation Through a Micropolar Slab Sandwiched by Two Elastic Half-Spaces
}

Article in Journal of vibration and acoustics · March 2016

DOI: $10.1115 / 1.4033198$

CITATIONS

2

3 authors, including:

\section{Peng Zhang}

University of Science and Technology Beijing 6 PUBLICATIONS 8 CITATIONS

SEE PROFILE
READS

41

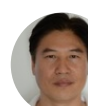

Peijun Wei

University of Science and Technology Beijing 72 PUBLICATIONS 280 CITATIONS

SEE PROFILE

Some of the authors of this publication are also working on these related projects: 
Department of Applied Mechanics,

University of Sciences and Technology Beijing,

Beijing 100083, China

Peijun Wei ${ }^{1}$

Department of Applied Mechanics,

University of Sciences and Technology Beijing,

Beijing 100083, China;

State Key Laboratory of Nonlinear

Mechanics (LNM),

Chinese Academy of Science,

Beijing 100080, China

e-mail: weipj@ustb.edu.cn

Yueqiu Li

Department of Applied Mechanics,

University of Sciences and Technology Beijing,

Beijing 100083, China by Two Elastic Half-Spaces

The problem of wave propagation through a micropolar elastic slab sandwiched by two classical elastic half-spaces is studied in this paper. Different from the classical elastic solids, the particle in micropolar solids can bear not only the displacements but also the rotations. The additional kinetic freedom results in four kinds of wave modes, namely, the longitudinal displacement (LD) wave, the longitudinal microrotational (LR) wave, and two coupled transverse (CT) waves. Apart from the $L D$ wave, the other three waves are dispersive. The existence of couple stresses and the microrotations also makes the interface conditions between the micropolar slab and the classic elastic half-spaces different from that between two classic solids. The nontraditional interface conditions lead to a set of algebraic equations from which the amplitude ratios of reflection and transmission waves can be determined. Further, the energy fluxes carried by various waves are evaluated and the energy conservation is checked to validate the numerical results obtained. The influences of the micropolar elastic constants and the thickness of slab are discussed based on the numerical results. Two situations of incident $P$ wave and incident $S H$ wave are both considered. [DOI: 10.1115/1.4033198]

Keywords: micropolar elasticity, sandwiched slab, reflection, transmission, energy flux

\section{Introduction}

In the problem of wave propagation, it is well known that the high-frequency elastic wave is of dispersive characteristic. However, the classical elastic theory predicts that the elastic waves are nondispersive. Therefore, the classic elastic theory is believed to be inadequate for a material possessing microstructure, in particular, when the wavelength of an incident wave is comparable to the length of the material microstructure. The composite that contains barlike elements and rigid elongated molecules, and fibrous materials such as wood and wood composites, some granular and porous solids, as well as some polymers, are extensively used in the passive noise control field in sound absorbers. In classical continuum mechanics, the effects of microstructure of a material are not taken into consideration. The evident discrepancies between the acoustic prediction obtained by classical theory and experimental results reveal the potential importance of the microstructure. In order to take the microstructure effects into consideration, the generalized continuum theories, for example, the nonlocal theory [1], the micromorphic theory [2], the couple stress theory $[3,4]$, the micropolar theory [5], and microstretch theory [6], were proposed successively. The micropolar elastic theory, as one of the high order elastic theory, is especially popular due to the clear mechanical concept compared with other high-order elastic theories. In the linear micropolar elastic theory developed by Eringen [5], each mass point has an extra microrotational degree-offreedom besides the translational ones. Accordingly, each mass point can suffer not only the force stresses but also the couple stresses. The additional kinetic degree-of-freedom results in four kinds of wave modes, namely, the LD wave, LR wave, and two CT waves (CT3 and CT4 waves). Apart from the LD wave, the other three waves are dispersive. Parfitt and Eringen [7] studied the reflection problem of micropolar waves from a free surface of a micropolar half-space and Ariman [8] studied the reflection problem from a fixed surface of a micropolar half-space and discussed some special cases of reflection waves. Tomar and Gogna

\footnotetext{
Corresponding author.

Contributed by the Technical Committee on Vibration and Sound of ASME for publication in the Journal OF VIBRATION AND Acoustics. Manuscript received September 1, 2015; final manuscript received March 8, 2016; published online May 23, 2016. Assoc. Editor: Michael Leamy.
}

[9,10] further studied the reflection and transmission problem of micropolar elastic waves at the interface between two different micropolar solids. Similar reflection and transmission problem from the interface between liquid and micropolar solid was studied by Tomar and Kumar [11]. Further, Singh [12,13] studied wave propagation in the generalized micropolar thermo-elastic solid and the anisotropic micropolar elastic solid. Khurana and Tomar $[14,15]$ studied the electric-mechanical coupling effects on the wave propagation in an electric-micropolar elastic solid. Moreover, Chiriţă and Ghiba [16] also studied the Rayleigh waves in a micropolar elastic solid. Kumari [17] studied the effects of an imperfect boundary between the micropolar viscoelastic solid and fluid saturated incompressible porous solid. Zhang et al. [18] studied effects of nonfree surface on the reflection waves in a micropolar solid. In all the above-mentioned investigations, only single interface is concerned. The bi-interface problem will be much more complicated due to the multiple reflection and transmission process between the two interfaces. Hsia and $\mathrm{Su}$ [19] studied the propagation of longitudinal waves in microporous slab sandwiched between elastic half-spaces. However, the interface conditions between the classic elastic solid and the micropolar solid are disputable and the numerical results are not validated by the energy conservation. The effects of thickness of slab are discussed only while the effects of micropolar constants are not discussed at all. Moreover, the dependence of reflection and transmission wave on the thickness-wavelength ratio should be discussed instead of the absolute thickness of slab.

In this paper, the reflection and transmission problem of an elastic wave through a micropolar slab sandwiched by two classic elastic half-spaces is studied. The micropolar elastic waves in the slab are divided into two groups, i.e., the forward and the backward waves, which are different for incident $P$ wave and incident $\mathrm{SH}$ wave. The interface conditions between the micropolar slab and the classic elastic half-spaces used by Hsia and Su [19] are modified, which includes the microrotation and the couple stresses, and are used to form a set of algebraic equations from which the amplitude ratios of various waves with respect to the incident wave are obtained. In order to validate the numerical results, the energy flux ratios of various waves with respect to the incident wave are further evaluated and the energy conservation is checked. The comparison between present work and that in 
literature [19] is performed, the deviations are analyzed and some mistakes in literature [19] are pointed out. Based on these numerical results, the influences of micropolar elastic constants and the nondimensional thickness of slab are discussed and some conclusions are obtained.

\section{Elastic Waves in the Micropolar Solids}

The motion equations of mass point in the micropolar elastic solid can be expressed as [7]

$$
\begin{array}{r}
(\lambda+2 \mu+\kappa) \nabla(\nabla \cdot \mathbf{u})-(\mu+\kappa) \nabla \times(\nabla \times \mathbf{u})+\kappa \nabla \times \boldsymbol{\varphi}=\rho \ddot{\mathbf{u}} \\
(\alpha+\beta+\gamma) \nabla(\nabla \cdot \boldsymbol{\varphi})-\gamma \nabla \times(\nabla \times \boldsymbol{\varphi})+\kappa \nabla \times \mathbf{u}-2 \kappa \boldsymbol{\varphi}=\rho j \ddot{\boldsymbol{\varphi}}
\end{array}
$$

where $\rho$ is the mass density and $j$ is the micro-inertia. $\mathbf{u}$ and $\boldsymbol{\varphi}$ are the displacement vector and the microrotation vector, respectively. Here, the body force and the body couple are ignored. The constitutive relation of micropolar solid can be expressed as

$$
\begin{gathered}
t_{k l}=\lambda u_{r, r} \delta_{k l}+\mu\left(u_{k, l}+u_{l, k}\right)+\kappa\left(u_{l, k}-\varepsilon_{k l r} \varphi_{r}\right) \\
m_{k l}=\alpha \varphi_{r, r} \delta_{k l}+\beta \varphi_{k, l}+\gamma \varphi_{l, k}
\end{gathered}
$$

where $t_{k l}$ and $m_{k l}$ are the force stress components and the couple stress components, respectively. $\lambda$ and $\mu$ are the classic lamé constants while $\kappa, \alpha, \beta$, and $\gamma$ are the micropolar elastic constants. Eringen [2] has found the inequalities between these material constants from the non-negative internal energy

$$
\begin{aligned}
& 0 \leq 3 \lambda+2 \mu+\kappa, \quad 0 \leq \mu, \quad 0 \leq \kappa, \quad 0 \leq 3 \alpha+\beta+\gamma, \\
& -\gamma \leq \beta \leq \gamma, \quad 0 \leq \gamma
\end{aligned}
$$

For purpose of uncoupling, we represent the $\mathbf{u}$ and $\varphi$ with a scalar potential and a vector potential

$$
\begin{array}{cc}
\mathbf{u}=\nabla q+\nabla \times \mathbf{U}, & \nabla \cdot \mathbf{U}=0 \\
\boldsymbol{\varphi}=\nabla \xi+\nabla \times \mathbf{\Phi}, & \nabla \cdot \boldsymbol{\Phi}=0
\end{array}
$$

Inserting Eq. (4) into Eq. (1) leads to

$$
\begin{gathered}
\left(c_{1}^{2}+c_{3}^{2}\right) \nabla^{2} q=\ddot{q} \\
\left(c_{4}^{2}+c_{5}^{2}\right) \nabla^{2} \xi-2 \omega_{0}^{2} \xi=\ddot{\xi} \\
\left(c_{2}^{2}+c_{3}^{2}\right) \nabla^{2} \mathbf{U}+c_{3}^{2} \nabla \times \boldsymbol{\Phi}=\ddot{\mathbf{U}} \\
c_{4}^{2} \nabla^{2} \boldsymbol{\Phi}+\omega_{0}^{2} \nabla \times \mathbf{U}-2 \omega_{0}^{2} \boldsymbol{\Phi}=\ddot{\boldsymbol{\Phi}}
\end{gathered}
$$

where $\quad c_{1}^{2}=(\lambda+2 \mu) / \rho, \quad c_{2}^{2}=\mu / \rho, \quad c_{3}^{2}=\kappa / \rho, \quad c_{4}^{2}=\gamma / \rho j$, $c_{5}^{2}=(\alpha+\beta) / \rho j, \omega_{0}^{2}=c_{3}^{2} / j=\kappa / \rho j$. The solution of Eq. (5) can be expressed as

$$
(q, \xi, \mathbf{U}, \mathbf{\Phi})=(a, b, \mathbf{A}, \mathbf{B}) \exp \left[i k_{p}\left(\mathbf{n}_{p} \cdot \mathbf{r}-v_{p} t\right)\right]
$$

where $a, b, \mathbf{A}$, and $\mathbf{B}$ are the complex amplitudes. $v_{p}$ is the phase velocity, $k_{p}$ is the wave number, $\mathbf{n}_{p}$ is the unit vector of propagation direction, and $\mathbf{r}$ is the position vector. $p=1,2,3,4$ stand for LD wave, LR wave, CT3 wave, and CT4 wave, respectively, which are explained as follows:

(a) An LD wave is created by the scalar field $q$. The phase speed is $v_{1}=\sqrt{(\lambda+2 \mu+\kappa) / \rho}$. It is a nondispersive wave. (b) An LR wave is created by the scalar field $\xi$. The phase speed is $v_{2}=\sqrt{(\alpha+\beta+\gamma) / \rho j\left(1-2 \kappa / \rho j \omega^{2}\right)}$. It is a dispersive wave.

(c) Two sets of coupled waves (CT3 and CT4) are created by the transverse displacement potential $\mathbf{U}$ and transverse microrotation potential $\boldsymbol{\Phi}$. These two sets of coupled waves are also dispersive and their phase speeds are

$$
v_{3,4}^{2}=\frac{1}{2(1-\varsigma)}\left[c_{4}^{2}+c_{3}^{2}+c_{2}^{2}-\left(c_{2}^{2}+c_{3}^{2} / 2\right) \varsigma \pm \Delta\right]
$$

where

$\Delta=\left\{\left[\left(c_{4}^{2}-c_{2}^{2}-c_{3}^{2}\right)+\left(c_{2}^{2}+c_{3}^{2} / 2\right) \varsigma\right]^{2}+2 c_{3}^{2} c_{4}^{2} \varsigma\right\}^{1 / 2}, \quad \varsigma=2 \omega_{0}^{2} / \omega^{2}$

It is noticed that $v_{2}$ and $v_{3}$ are real value only when $\omega>\sqrt{2} \omega_{0}$. Therefore, $\omega_{c}=\sqrt{2} \omega_{0}$ is the cutoff frequency of the LR wave and CT3 wave. It should be pointed out that the amplitude vectors $\mathbf{A}$ and $\mathbf{B}$ are perpendicular to each other and both orthogonal to the propagation direction $\mathbf{n}$ of the coupled waves. The explicit relation between them is

$$
\mathbf{B}=-\frac{i \omega_{0}^{2}}{k\left(v^{2}-2 \omega_{0}^{2} / k^{2}-c_{4}^{2}\right)} \mathbf{n} \times \mathbf{A}
$$

In the plane strain situation, $u_{1} \neq 0, u_{3} \neq 0, u_{2}=0$ and $\varphi_{1}=0, \varphi_{3}=0, \varphi_{2} \neq 0$. Then, $q(x, z) \neq 0, \xi(x, z)=0, \mathbf{U}(x, z)=U_{2} \mathbf{e}_{2}$, and $\boldsymbol{\Phi}(x, z)=\Phi_{1} \mathbf{e}_{1}+\Phi_{3} \mathbf{e}_{3}$. In the out-of-plane strain situation, $u_{1}=0, u_{3}=0, u_{2} \neq 0$ and $\varphi_{1} \neq 0, \varphi_{3} \neq 0, \varphi_{2}=0$. Then, $q(x, z)=0$, $\xi(x, z) \neq 0, \quad \mathbf{U}(x, z)=U_{1} \mathbf{e}_{1}+U_{3} \mathbf{e}_{3}$ and $\boldsymbol{\Phi}(x, z)=\Phi_{2} \mathbf{e}_{2}$. In other words, LR wave does not exist for plane strain case while LD wave does not exist for out-of-plane strain case. In the present work, the two cases are both considered.

\section{Reflection and Transmission Through a Micropolar Slab}

3.1 Incident $\mathbf{P}$ Wave Situation. Considering a micropolar slab of thickness $h$ sandwiched by two elastic half-spaces, see Fig. 1. Medium $1(z<0)$ and medium $3(z>h)$ are assumed to be same classic elastic solids and characterized by lamé coefficients $\lambda_{1}$ and $\mu_{1}$. Medium 2 is a micropolar slab characterized by lamé coefficients $\lambda_{2}, \mu_{2}$ and the micropolar elastic constants $\left(\kappa_{2}, \alpha_{2}, \beta_{2}, \gamma_{2}, j_{2}\right)$. Incident $P$ wave is from medium 1 . And results in the reflection $P$ and $S V$ waves in medium 1 while the transmission $P$ and $S V$ waves in medium 3. In the micropolar slab, there

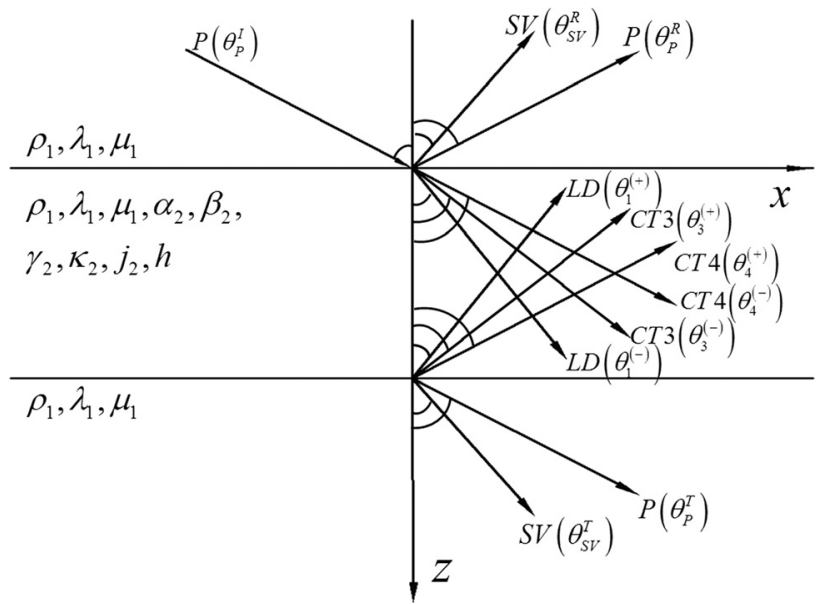

Fig. 1 Wave propagation through a micropolar slab sandwiched by two elastic half-spaces 
are a set of forward waves $\left(\mathrm{LD}^{(+)}, \mathrm{CT} 3^{(+)}, \mathrm{CT} 4^{(+)}\right)$and a set of backward waves $\left(\mathrm{LD}^{(-)}, \mathrm{CT} 3^{(-)}, \mathrm{CT} 4^{(-)}\right)$induced by the incident wave, see Fig. 1. The incident wave, the reflection waves and the transmission waves in classical elastic media, and the forward and backward waves in micropolar slab, can be explicitly expressed in terms of potential functions and given in Appendix A. The displacement, force stress, and couple stress components in the micropolar slab can be obtained by substituting Eq. (6) into Eqs. (2) and (4), namely,

$$
\begin{gathered}
u_{1}^{2}=q_{, x}^{(+)}+q_{, x}^{(-)}-U_{2, z}^{(+)}-U_{2, z}^{(-)} \\
u_{3}^{2}=q_{, z}^{(+)}+q_{, z}^{(-)}+U_{2, x}^{(+)}+U_{2, x}^{(+)} \\
t_{z z}^{2}=\left(\lambda_{2}+2 \mu_{2}+\kappa_{2}\right)\left(q_{, z z}^{(+)}+q_{, z z}^{(-)}\right)+\lambda_{2}\left(q_{, x x}^{(+)}+q_{x x}^{(-)}\right) \\
+\left(2 \mu_{2}+\kappa_{2}\right)\left(U_{2, x z}^{(+)}+U_{2, x z}^{(-)}\right) \\
t_{z x}^{2}=\left(2 \mu_{2}+\kappa_{2}\right)\left(q_{, x z}^{(+)}+q_{, x z}^{(-)}\right)-\left(\mu_{2}+\kappa_{2}\right)\left(U_{2, z z}^{(+)}+U_{2, z z}^{(-)}\right) \\
+\mu_{2}\left(U_{2, x x}^{(+)}+U_{2, x x}^{(-)}\right)-\kappa_{2}\left(\varphi_{2}^{(+)}+\varphi_{2}^{(-)}\right) \\
m_{z y}^{2}=\gamma_{2}\left(\varphi_{2, z}^{(+)}+\varphi_{2, z}^{(-)}\right)
\end{gathered}
$$

The displacement and force stress components in medium 1 are

$$
\begin{gathered}
u_{1}^{1}=q_{, x}^{I}+q_{, x}^{R}-U_{2, z}^{R} \\
u_{3}^{1}=q_{, z}^{I}+q_{, z}^{R}+U_{2, x}^{R} \\
t_{z z}^{1}=\left(\lambda_{1}+2 \mu_{1}\right)\left(q_{, z z}^{I}+q_{, z z}^{R}\right)+\lambda_{1}\left(q_{, x x}^{I}+q_{, x x}^{R}\right)+2 \mu_{1} U_{2, x z}^{R} \\
t_{z x}^{1}=2 \mu_{1}\left(q_{x z}^{I}+q_{, x z}^{R}\right)+\mu_{1}\left(U_{2, x x}^{R}-U_{2, z z}^{R}\right)
\end{gathered}
$$

And the displacement and force stress components in medium 3 are

$$
\begin{gathered}
u_{1}^{3}=q_{, x}^{T}-U_{2, z}^{T} \\
u_{3}^{3}=q_{, z}^{T}+U_{2, x}^{T} \\
t_{z z}^{3}=\left(\lambda_{1}+2 \mu_{1}\right) q_{, z z}^{T}+\lambda_{1} q_{x x}^{T}+2 \mu_{1} U_{2, x z}^{T} \\
t_{z x}^{3}=2 \mu_{1} q_{x z}^{T}+\mu_{1}\left(U_{2, x x}^{T}-U_{2, z z}^{T}\right)
\end{gathered}
$$

Because the classic elastic solid cannot suffer the couple stress and the microrotation, the interface conditions at the interfaces $z=0$ and $z=h$, can be written in two forms, namely,

Form I

$t_{z z}^{1}=t_{z z}^{2}, \quad t_{z x}^{1}=t_{z x}^{2}, \quad u_{x}^{1}=u_{x}^{2}, \quad u_{z}^{1}=u_{z}^{2}, \quad m_{z y}^{2}=0 . \quad(z=0)$

$t_{z z}^{2}=t_{z z}^{3}, \quad t_{z x}^{2}=t_{z x}^{3}, \quad u_{x}^{2}=u_{x}^{3}, \quad u_{z}^{2}=u_{z}^{3}, \quad m_{z y}^{2}=0 . \quad(z=h)$

Form II

$$
t_{z z}^{1}=t_{z z}^{2}, \quad t_{z x}^{1}=t_{z x}^{2}, \quad u_{x}^{1}=u_{x}^{2}, \quad u_{z}^{1}=u_{z}^{2}, \quad \varphi_{2}^{2}=0 . \quad(z=0)
$$

$$
t_{z z}^{2}=t_{z z}^{3}, \quad t_{z x}^{2}=t_{z x}^{3}, \quad u_{x}^{2}=u_{x}^{3}, \quad u_{z}^{2}=u_{z}^{3}, \quad \varphi_{2}^{2}=0 . \quad(z=h)
$$

The more appropriate interface condition about the couple stress and the microrotation should be expressed as

$$
d m_{z y}^{2}+(1-d) K_{y} \varphi_{2}^{2}=0 \quad(z=0, h) \quad \text { and } \quad d \in(0,1)
$$

where $K_{y}$ is scaling factor which is chosen to make the two terms at the left side of Eq. (13) having same order of magnitudes and same dimension; $d$ is the weighed factor. Equation (13) reduces to the form I and form II as $d=1$ and $d=0$, respectively. From these interface conditions, it is deduced that the various waves have same angular frequency and the same apparent wavenumber with the incident wave, namely,

$$
\omega_{i}^{j}=\omega_{P}^{I}, \quad \frac{\sin \theta_{i}^{j}}{v_{i}}=\frac{\sin \theta_{P}^{I}}{v_{P}}, \quad(i=P, S V, 1,3,4, j=I, R, T,(+),(-))
$$

The amplitude ratios of reflection waves and transmission waves with respect to the incident wave satisfy the following algebraic equations:

$$
\mathbf{A}_{P} \mathbf{Z}_{P}=\mathbf{B}_{P}
$$

where

$$
\mathbf{Z}_{P}=\left[\left(a^{R}, A^{R}, a^{(+)}, A_{3}^{(+)}, A_{4}^{(+)}, a^{(-)}, A_{3}^{(-)}, A_{4}^{(-)}, a^{T}, A^{T}\right) / a^{I}\right]^{T}
$$

The explicit expressions of the elements of matrices $\mathbf{A}_{P}$ and $\mathbf{B}_{P}$ in Eq. (15) are listed in Appendix B.

3.2 Incident SH Wave Situation. In the case of incident $S H$ wave, the incident wave, the reflection waves and the transmission waves in classical elastic media, and the forward and backward waves in micropolar slab, can also be expressed in terms of potential functions and given in Appendix A. The microrotation, force stress, and the couple stress components in the micropolar slab are

$$
\begin{gathered}
\varphi_{x}^{2}=\left(\xi_{, x}^{(+)}+\xi_{, x}^{(-)}\right)-\left(\Phi_{2, z}^{(+)}+\Phi_{2, z}^{(-)}\right) \\
\varphi_{z}^{2}=\left(\xi_{, z}^{(+)}+\xi_{, z}^{(-)}\right)+\left(\Phi_{2, x}^{(+)}+\Phi_{2, x}^{(-)}\right) \\
t_{z y}^{2}=\left(\mu_{2}+\kappa_{2}\right)\left(u_{2, z}^{(+)}+u_{2, z}^{(-)}\right)+\kappa_{2}\left[\left(\xi_{, x}^{(+)}+\xi_{, x}^{(-)}\right)-\left(\Phi_{2, z}^{(+)}+\Phi_{2, z}^{(-)}\right)\right] \\
m_{z x}^{2}=\left(\beta_{2}+\gamma_{2}\right)\left(\xi_{, x z}^{(+)}+\xi_{, x z}^{(-)}\right)+\beta_{2}\left(\Phi_{2, x x}^{(+)}+\Phi_{2, x x}^{(-)}\right) \\
-\gamma_{2}\left(\Phi_{2, z z}^{(+)}+\Phi_{2, z z}^{(-)}\right) \\
m_{z z}^{2}=\alpha_{2}\left[\left(\xi_{, x x}^{(+)}+\xi_{, x x}^{(-)}\right)+\left(\xi_{, z z}^{(+)}+\xi_{, z z}^{(-)}\right)\right] \\
+\left(\beta_{2}+\gamma_{2}\right)\left[\left(\xi_{, z z}^{(+)}+\xi_{, z z}^{(-)}\right)+\left(\Phi_{2, x z}^{(+)}+\Phi_{2, x z}^{(-)}\right)\right]
\end{gathered}
$$

The force stress components in medium 1 and medium 3 are

$$
\begin{gathered}
t_{z y}^{1}=\mu_{1}\left(u_{2, x z}^{I}+u_{2, x z}^{R}\right) \\
t_{z y}^{3}=\mu_{1} u_{2, x z}^{T}
\end{gathered}
$$

The interface conditions at the interfaces $z=0$ and $z=h$ can be expressed as 


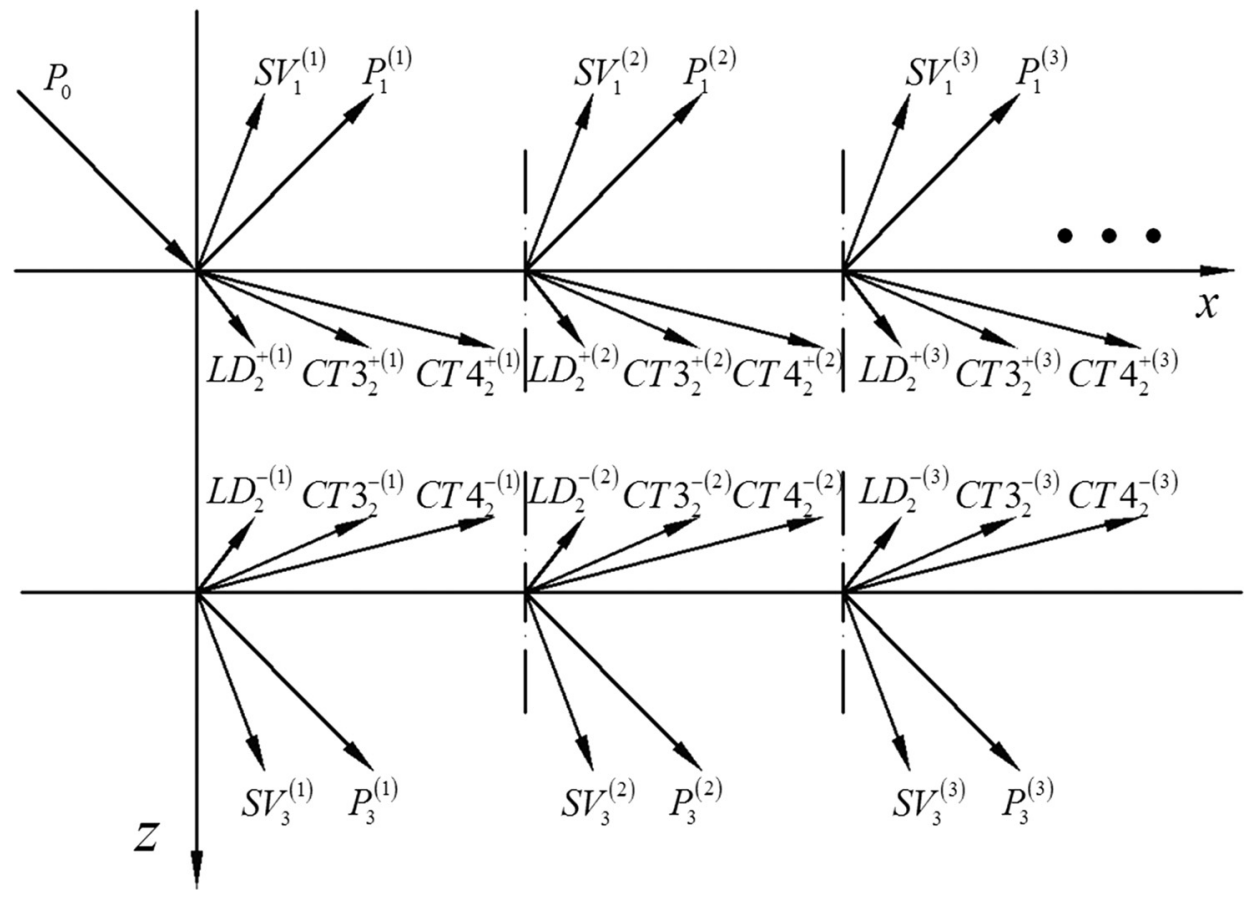

Fig. 2 Sketch of multiple reflection and transmission waves
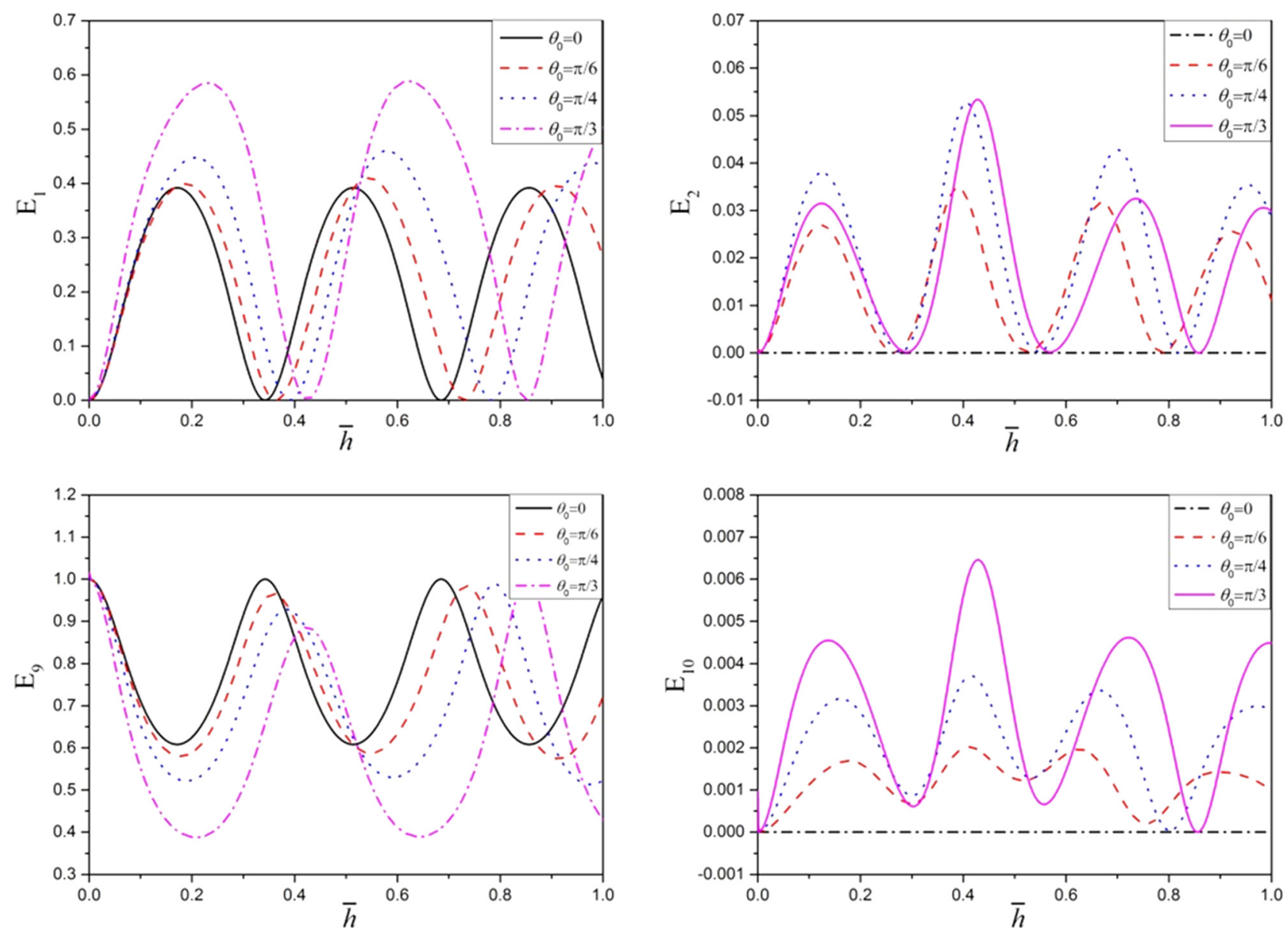

Fig. 3 The dependence of the reflection and transmission coefficients upon nondimensional thickness $\bar{h}\left(=h / L_{0}\right)$ in the case of incident $P$ wave $\left(d=0, \bar{\mu}_{1}=0.2, \bar{\lambda}_{2}=0.2, \bar{\mu}_{2}=0.1, \bar{\rho}_{2}=0.7, \bar{\gamma}_{2}=0.1, \bar{\kappa}_{2}=0.06, \bar{j}_{2}=1\right)$

041008-4 / Vol. 138, AUGUST 2016

Transactions of the ASME 


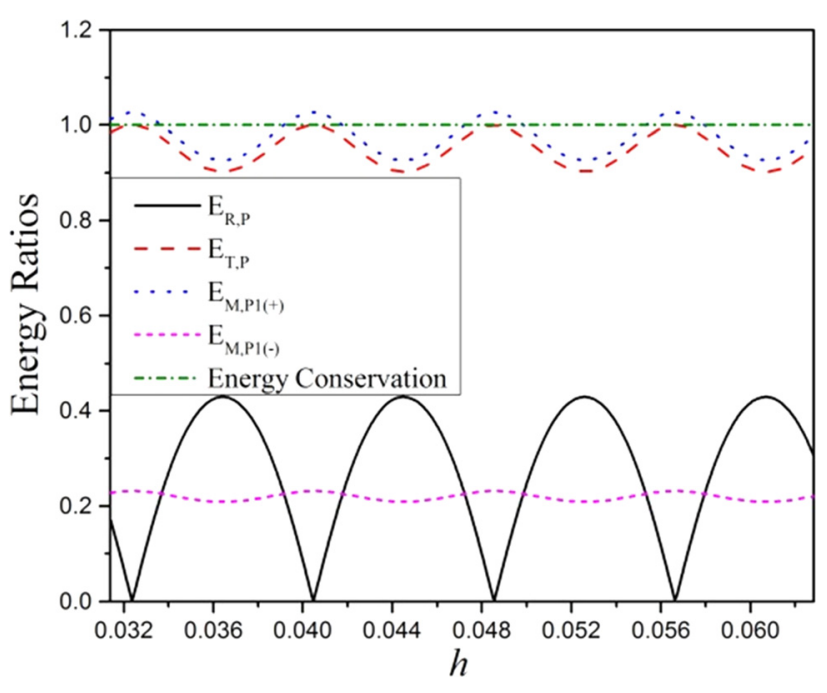

Fig. 4 The dependence of energy ratios on the thickness of micropolar sandwiched layer in the case of normal incident $P$ wave at $0.5 \mathrm{MHz}$ (here, the energy ratios are modified to keep consistence with that in literature [19])

$$
\begin{gathered}
t_{z y}^{1}=t_{z y}^{2}, \quad u_{2}^{1}=u_{2}^{2}, \quad d m_{z x}^{2}+(1-d) K_{x} \varphi_{x}^{2}=0, \\
d m_{z z}^{2}+(1-d) K_{z} \varphi_{z}^{2}=0 . \quad(z=0)
\end{gathered}
$$
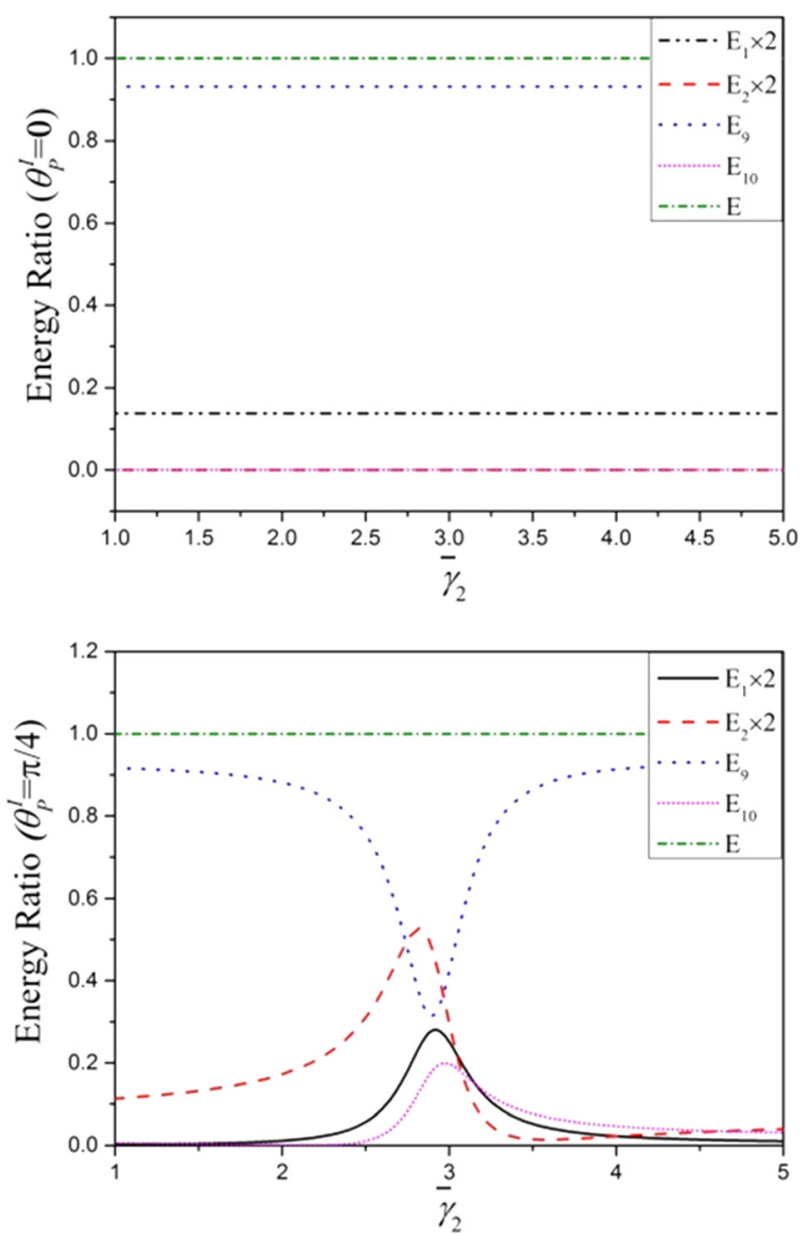

$$
\begin{gathered}
t_{z y}^{2}=t_{z y}^{3}, \quad u_{2}^{2}=u_{2}^{3}, \quad d m_{z x}^{2}+(1-d) K_{x} \varphi_{x}^{2}=0, \\
d m_{z z}^{2}+(1-d) K_{z} \varphi_{z}^{2}=0 . \quad(z=h)
\end{gathered}
$$

where $K_{x}$ and $K_{z}$ are the scaling factors which are similar with $K_{y}$ in Eq. (13). These interface conditions require that various waves have same angular frequency and the same apparent wavenumber, namely,

$$
\omega_{i}^{j}=\omega_{P}^{I}, \frac{\sin \theta_{i}^{j}}{v_{i}}=\frac{\sin \theta_{S H}^{I}}{v_{S H}}, \quad(i=S H, 1,3,4, j=I, R, T,(+),(-))
$$

The amplitude ratios of various waves with respect to the incident wave satisfy the algebraic equations

$$
\mathbf{A}_{S} \mathbf{Z}_{S}=\mathbf{B}_{S}
$$

where

$$
\mathbf{Z}_{S}=\left[\left(B^{R}, b^{(+)}, B_{3}^{(+)}, B_{4}^{(+)}, b^{(-)}, B_{3}^{(-)}, B_{4}^{(-)}, B^{T}\right) / B^{I}\right]^{T}
$$

The explicit expressions of the elements of matrices $\mathbf{A}_{S}$ and $\mathbf{B}_{S}$ in Eq. (21) are listed in Appendix B.

3.3 Multiple Reflection and Transmission Approach. Although the amplitude ratios of reflection and transmission waves can be obtained from the interface conditions, the propagation process through a slab and the formation of resulting
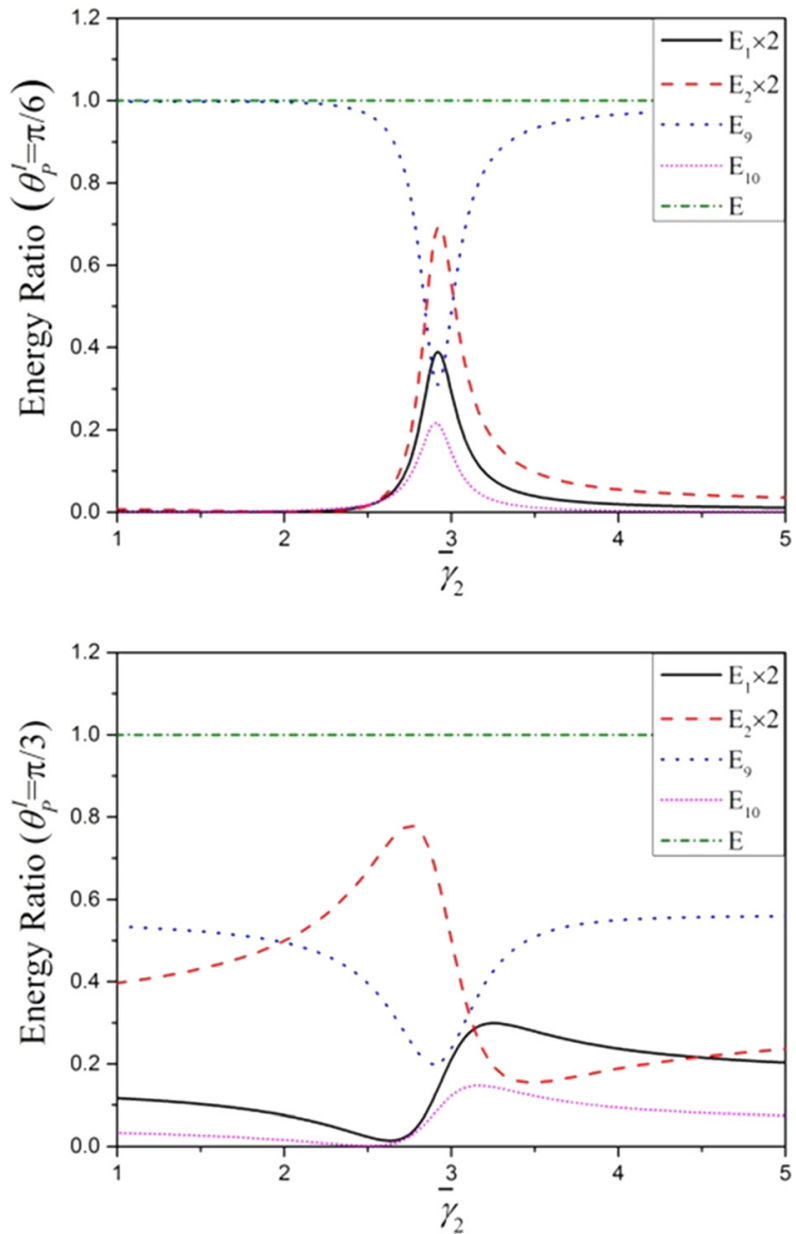

Fig. 5 The dependence of the reflection and transmission coefficients upon the micropolar constant $\bar{\gamma}_{2}$ for different incident angles $\theta_{P}^{\prime}$ in the case of incident $P$ wave $\left(d=0, \bar{\mu}_{1}=0.75, \bar{\lambda}_{2}=0.8, \bar{\mu}_{2}=0.6, \bar{\rho}_{2}=1.2, \bar{\kappa}_{2}=6, \bar{j}_{2}=30, \bar{h}=0.1\right)$ 

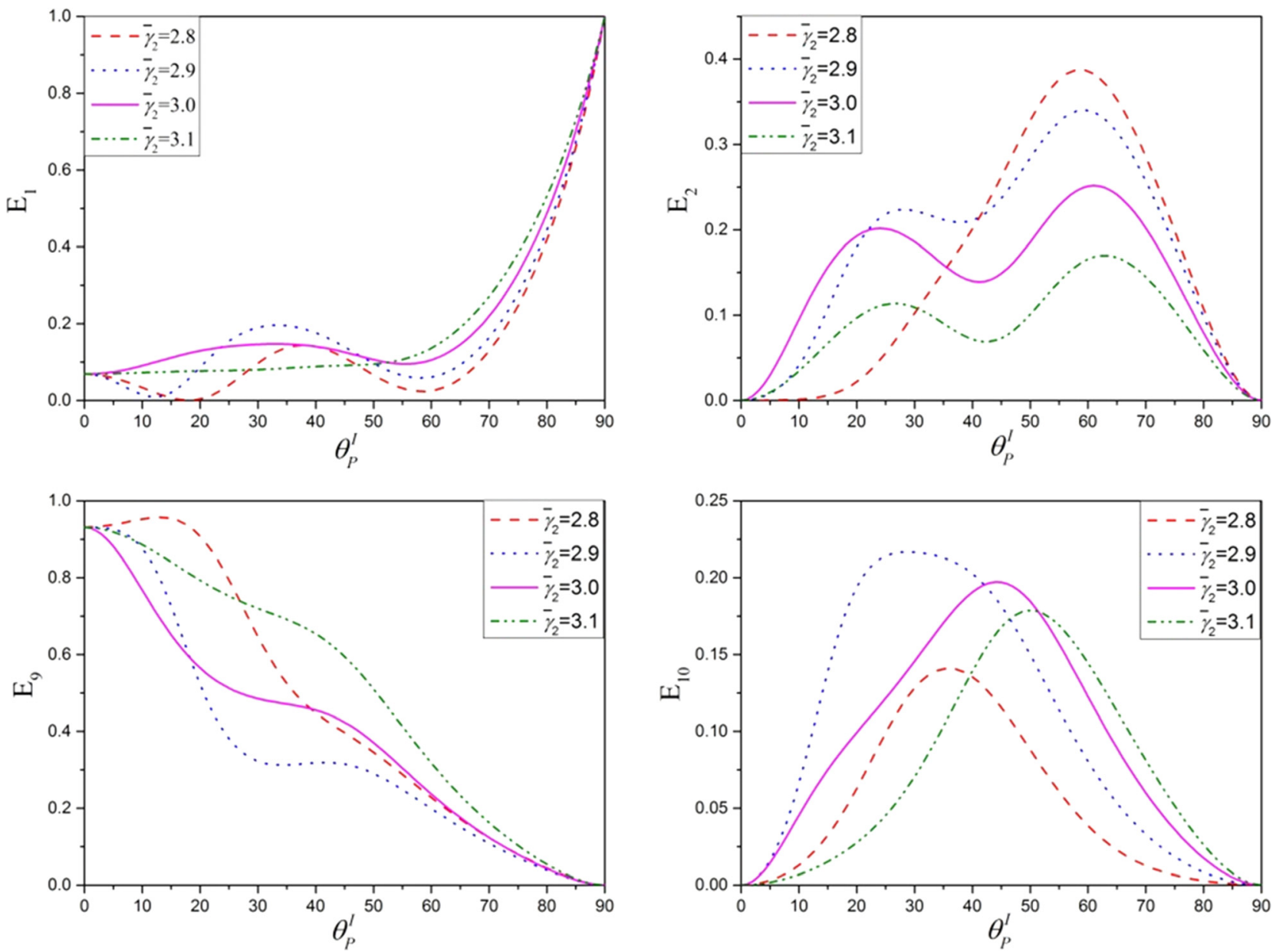

Fig. 6 The dependence of the reflection and transmission coefficients upon the incident angle $\theta_{P}^{I}$ for different micropolar constants $\bar{\gamma}_{2}$ in the case of incident $P$ wave $\left(d=0, \bar{\mu}_{1}=0.75, \bar{\lambda}_{2}=0.8, \bar{\mu}_{2}=0.6, \bar{\rho}_{2}=1.2, \bar{\kappa}_{2}=6, \bar{j}_{2}=30, \bar{h}=0.1\right)$

reflection and transmission waves are concealed. In order to present a clearer physical picture of wave propagation through a slab and the formation of resulting reflection and transmission waves, we appeal to the multiple reflection and transmission approach.

In the case of incident $P$ or incident $S V$ wave, the matrix formulation is necessary for the multiple reflection and transmission process due to the coupled nature between the longitudinal wave and the transverse wave. Let $\mathbf{H}^{\mathrm{I}}=\left(A^{I}, B^{I}\right)^{T}, \mathbf{H}^{\mathrm{R}}=\left(A^{R}, B^{R}\right)^{T}$, and $\mathbf{H}^{\mathrm{T}}=\left(A^{T}, B^{T}\right)^{T}$ represent the amplitude vectors of the incident waves, the reflection waves, and the transmission waves, respectively. $\mathbf{R}_{i j}^{P}$ and $\mathbf{T}_{i j}^{P}$ are the reflection and transmission matrices at the interface between medium $i$ and medium $j$ when incident wave is from medium $i$. Then, the resultant reflection and transmission waves can be expressed as

$\mathbf{H}^{\mathrm{R}}=\mathbf{R}_{12}^{P} \mathbf{H}^{\mathrm{I}}+\mathbf{T}_{21}^{P} \boldsymbol{\Lambda} \mathbf{R}_{23}^{P} \mathbf{\Lambda} \mathbf{T}_{12}^{P} \mathbf{H}^{\mathrm{I}}+\mathbf{T}_{21}^{P} \boldsymbol{\Lambda} \mathbf{R}_{23}^{P} \mathbf{\Lambda} \mathbf{R}_{21}^{P} \mathbf{\Lambda} \mathbf{R}_{23}^{P} \mathbf{\Lambda} \mathbf{T}_{12}^{P} \mathbf{H}^{\mathrm{I}}+\cdots$

$$
\begin{aligned}
\mathbf{H}^{\mathrm{T}}= & \mathbf{T}_{23}^{P} \boldsymbol{\Lambda} \mathbf{T}_{12}^{P} \mathbf{H}^{\mathrm{I}}+\mathbf{T}_{23}^{P} \boldsymbol{\Lambda} \mathbf{R}_{21}^{P} \boldsymbol{\Lambda} \mathbf{R}_{23}^{P} \boldsymbol{\Lambda} \mathbf{T}_{12}^{P} \mathbf{H}^{\mathrm{I}} \\
& +\mathbf{T}_{23}^{P} \boldsymbol{\Lambda} \mathbf{R}_{21}^{P} \boldsymbol{\Lambda} \mathbf{R}_{23}^{P} \boldsymbol{\Lambda} \mathbf{R}_{21}^{P} \boldsymbol{\Lambda} \mathbf{R}_{23}^{P} \boldsymbol{\Lambda} \mathbf{T}_{12}^{P} \mathbf{H}^{\mathrm{I}}+\cdots
\end{aligned}
$$

where

$$
\boldsymbol{\Lambda}=\left(\begin{array}{ccc}
\exp \left(i k_{y}^{\mathrm{LD}} h\right) & 0 & 0 \\
0 & \exp \left(i k_{y}^{\mathrm{CT} 3} h\right) & 0 \\
0 & 0 & \exp \left(i k_{y}^{\mathrm{CT} 4} h\right)
\end{array}\right)
$$

is the phase shift matrix of micropolar elastic waves which propagates for one-way trip between the interface I $(z=0)$ and the interface II $(z=h)$. On the right side of Eq. $(22 a)$, the first term stands for the first reflection wave at interface I. The second term stands for the second reflection wave. It is created by experiencing the transmission first across interface I, then propagates from interface I to interface II, and reflects at interface II, returns back to interface I, and finally transmits through interface I into medium 1 . The second reflection wave makes a round trip between interface I and interface II. The third reflection wave makes double round trips and so on. The summation of these multiple reflection waves leads to the resultant reflection waves in medium 1. Similarly, the first term in Eq. (22b) stands for the first transmission wave; the second term the second transmission wave, and so on. The summation of these multiple transmission waves leads to the resultant transmission waves in medium 3 . The multiple reflection and transmission process is shown in Fig. 2. After performing the summation of infinite series of matrices, Eq. (22) becomes

$$
\begin{gathered}
\mathbf{H}^{\mathrm{R}}=\left[\mathbf{R}_{12}^{P}+\mathbf{T}_{21}^{P}\left(\mathbf{I}-\boldsymbol{\Lambda} \mathbf{R}_{23}^{P} \boldsymbol{\Lambda} \mathbf{R}_{21}^{P}\right)^{-1} \boldsymbol{\Lambda} \mathbf{R}_{23}^{P} \boldsymbol{\Lambda} \mathbf{T}_{12}^{P}\right] \mathbf{H}^{\mathrm{I}} \\
\mathbf{H}^{\mathrm{T}}=\mathbf{T}_{23}^{P}\left(\mathbf{I}-\boldsymbol{\Lambda} \mathbf{R}_{21}^{P} \boldsymbol{\Lambda} \mathbf{R}_{23}^{P}\right)^{-1} \boldsymbol{\Lambda} \mathbf{T}_{12}^{P} \mathbf{H}^{\mathrm{I}}
\end{gathered}
$$

Equation (23) implies that the reflection and transmission coefficients are the period function of the thickness $k_{y} h$. The multiple reflection and transmission waves for incident $\mathrm{SH}$ wave situation can be analyzed similarly. 

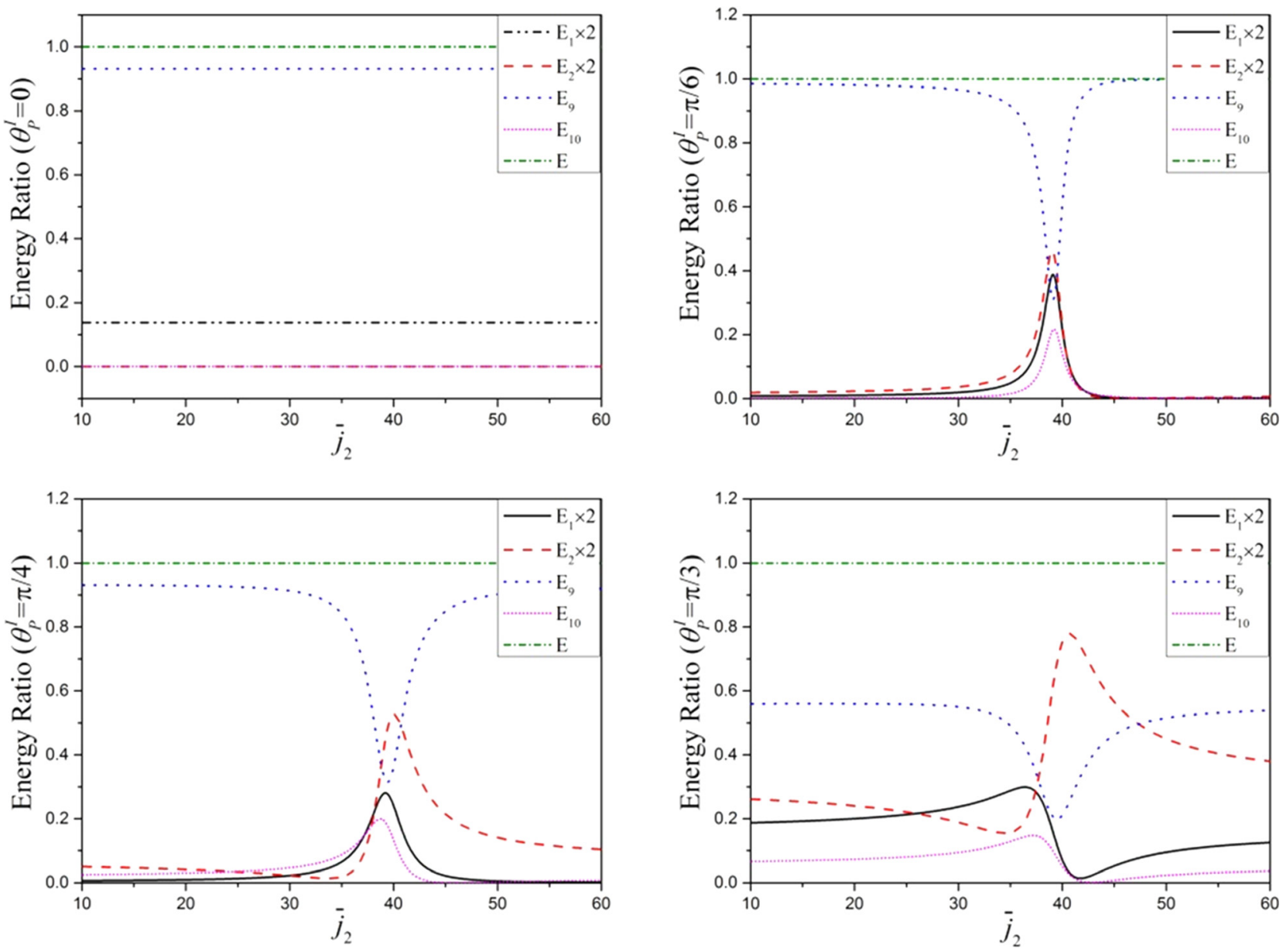

Fig. 7 The dependence of the reflection and transmission coefficients upon the micro-inertia $\bar{j}_{2}$ for different incident angles $\theta_{P}^{\prime}$ in the case of incident $P$ wave $\left(d=0, \bar{\mu}_{1}=0.75, \bar{\lambda}_{2}=0.8, \bar{\mu}_{2}=0.6, \bar{\rho}_{2}=1.2, \bar{\gamma}_{2}=4, \bar{\kappa}_{2}=6, \bar{h}=0.1\right)$

3.4 Limiting Cases. It is observed that the micropolar solid will degenerate into classical elastic solid when the micropolar constants $\kappa, \alpha, \beta, \gamma$ equal to zero. In the degeneration, the LD wave turns into $P$ wave, the CT3 wave turns into $S V$ wave (in the case of plane strain) or $S H$ wave (in the case of antiplane strain) and other waves vanish.

\section{Energy Flux Partition and the Energy Conservation}

The energy flux carried by a beam of wave can be estimated by

$$
P_{i}(t)=-\left[\operatorname{Re}\left(\dot{u}_{j}\right) \operatorname{Re}\left(t_{i j}\right)+\operatorname{Re}\left(\dot{\varphi}_{j}\right) \operatorname{Re}\left(m_{i j}\right)\right]
$$

where the first term is due to the force stress while the second term is due to the couple stress. For the reflection and transmission waves in the classic elastic solids, the second term in Eq. (24) can be ignored. However, it is not ignored any more in the present situation when the effects of microstructure are taken into consideration. The average energy flux in one period is

$$
\overline{P_{i}}=\frac{\omega}{2 \pi} \int_{-\pi / \omega}^{\pi / \omega} P_{i}(t) d t=-\frac{1}{2} \operatorname{Re}\left(\dot{u}_{j}^{*} t_{i j}+\dot{\varphi}_{j}^{*} m_{i j}\right)
$$

where superscript “*” indicates the complex conjugate. The energy flux along the propagation direction $\mathbf{n}$ is

$$
\overline{P_{n}}(\mathbf{n})=\overline{P_{x}} \cos (\mathbf{n}, \mathbf{x})+\overline{P_{z}} \cos (\mathbf{n}, \mathbf{z})
$$

where $\overline{P_{x}}$ and $\overline{P_{z}}$ are the energy fluxes along the $x$ and $z$ directions. The reflection and transmission coefficients are defined as the ratios of energy fluxes of the reflected and transmission waves with respect to the incident wave, namely,

$$
E_{i}=\frac{\overline{P_{n}}\left(\mathbf{n}_{i}\right)}{\overline{P_{n}^{I}}\left(\mathbf{n}_{0}\right)} ; \quad(i=1,2,3, \ldots, X)
$$

where $\overline{P_{n}^{I}}\left(\mathbf{n}_{0}\right)$ is the energy flux of incident wave, $\overline{P_{n}}\left(\mathbf{n}_{i}\right)$ are the energy fluxes of reflected and transmission waves. In the case of incident $P$ wave $(X=10), i=1,2$ stand for reflected $P$ and $S V$ waves in medium $1, i=3,4,5$ stand for the forward $\mathrm{LD}$, CT3, and CT4 waves in medium $2, i=6,7,8$ stand for the backward LD, CT3, and CT4 waves in medium 2, $i=9,10$ stand for transmission $P$ and $S V$ waves in medium 3 . In the case of incident $S H$ wave $(X=8), i=1$ stands for reflected $S H$ wave in medium $1, i=2,3,4$ stand for forward LR, CT3, and CT4 waves in medium 2, $i=5,6,7$ stand for backward LR, CT3, and CT4 waves in medium $2, i=8$ stands for transmission $S H$ wave in medium 3 .

The energy conservation at the interface requires that the input energy flux at unit area at the interface is equal to the output energy fluxes from the same area. In the case of incident $P$ wave

$$
\begin{aligned}
& \overline{P_{z P}^{I}}-\overline{P_{z \mathrm{LD}}^{(-)}}-\overline{P_{z \mathrm{CT} 3}^{(-)}}-\overline{P_{z \mathrm{CT} 4}^{(-)}}=-\overline{P_{z P}^{R}}-\overline{P_{z S V}^{R}}+\overline{P_{z \mathrm{LD}}^{(+)}}+\overline{P_{z \mathrm{CT} 3}^{(+)}} \\
&+\overline{P_{z \mathrm{CT} 4}^{(+)}}, \quad(z=0) \\
& \overline{P_{\mathrm{zLD}}^{(+)}}+\overline{P_{z \mathrm{CT} 3}^{(+)}}+\overline{P_{z \mathrm{CT} 4}^{(+)}}=\overline{P_{z P}^{T}}+\overline{P_{z S V}^{T}}-\overline{P_{z \mathrm{LD}}^{(-)}}-\overline{P_{z \mathrm{CT} 3}^{(-)}}-\overline{P_{z \mathrm{CT} 4}^{(-)}}, \quad(2=h)
\end{aligned}
$$



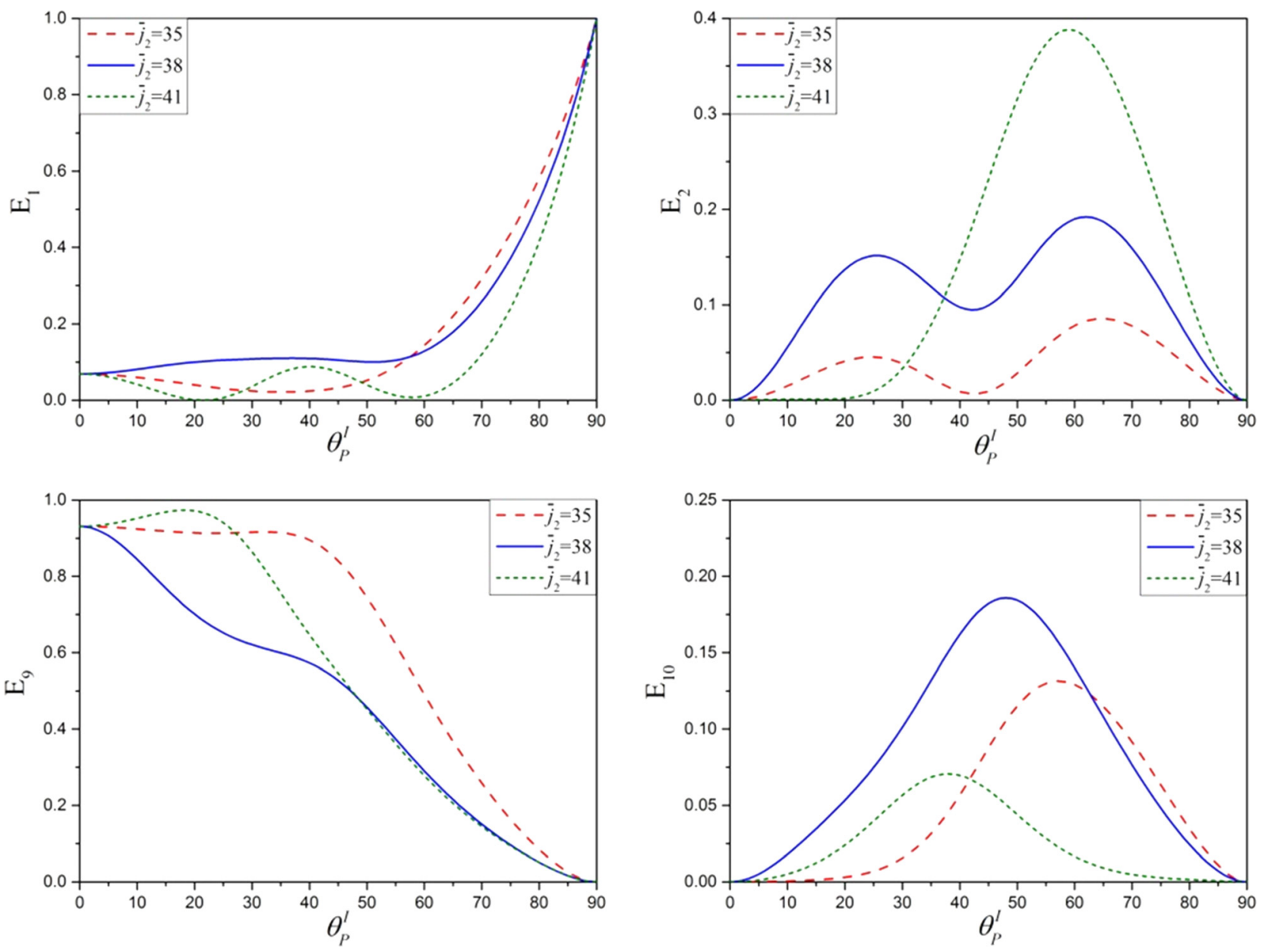

Fig. 8 The dependence of the reflection and transmission coefficients upon the incident angle $\theta_{P}^{I}$ for different micro-inertia $\bar{j}_{2}$ in the case of incident $P$ wave $\left(d=0, \bar{\mu}_{1}=0.75, \bar{\lambda}_{2}=0.8, \bar{\mu}_{2}=0.6, \bar{\rho}_{2}=1.2, \bar{\gamma}_{2}=4, \bar{\kappa}_{2}=6, \bar{h}=0.1\right)$

Combing Eqs. (28a) and (28b) leads to

$$
\overline{P_{z P}^{I}}=-\overline{P_{z P}^{R}}-\overline{P_{z S V}^{R}}+\overline{P_{z P}^{T}}+\overline{P_{z S V}^{T}}
$$

which signifies that the micropolar slab does not absorb energy and the energy carried by incident wave are completely assigned to the reflection waves and the transmission waves. By the introduction of energy conservation index E, Eq. (29) can be rewritten as

$$
E=-\frac{\overline{P_{z P}^{R}}}{\overline{P_{z P}^{I}}}-\frac{\overline{P_{z S V}^{R}}}{\overline{P_{z P}^{I}}}+\frac{\overline{P_{z P}^{T}}}{\overline{P_{z P}^{I}}}+\frac{\overline{P_{z S V}^{T}}}{\overline{P_{z P}^{I}}}=1
$$

Similarly, in the case of incident $S H$ wave, the energy conservation requires

$$
E=-\frac{\overline{P_{z S H}^{R}}}{\overline{P_{z S H}^{I}}}+\frac{\overline{P_{z S H}^{T}}}{\overline{P_{z S H}^{I}}}=1
$$

Equations (30) and (31) are used to validate the numerical results in Sec. 5.

\section{Numerical Results and Discussion}

It is known that the reflection and transmission of waves are only dependent on the incident wave when medium 1, medium 2 , and medium 3 are prescribed. For convenience sake, medium 1 and medium 3 are assumed to be identical. Then, the dependence relation between the reflection/transmission waves and the incident wave can be written as

$$
\left(A_{i}, a_{i}, B_{i}, b_{i}\right)=f\left(\lambda_{1}, \mu_{1}, \rho_{1}, \lambda_{2}, \mu_{2}, \rho_{2}, \alpha_{2}, \beta_{2}, \gamma_{2}, \kappa_{2}, j_{2}, h, A_{0}, L_{0}, \omega_{0}, \theta_{0}\right)
$$

where $\left(\lambda_{1}, \mu_{1}, \rho_{1}\right)$ are the elastic constants and the mass density of medium 1. $\left(\lambda_{2}, \mu_{2}, \rho_{2}, \alpha_{2}, \beta_{2}, \gamma_{2}, \kappa_{2}, j_{2}, h\right)$ are the material constants of micropolar slab. $\left(A_{0}, L_{0}, \omega_{0}, \theta_{0}\right)$ are the parameters of incident wave, namely, the amplitude $A_{0}$, the wavelength $L_{0}$, the angular frequency $\omega_{0}$ and the incident angle $\theta_{0}$. Choosing $\left(\rho_{1}, \omega_{0}, L_{0}\right)$ as the basic physical quantities, then, the nondimensional form of Eq. (32) can be written as

$$
\left(A_{i}, a_{i}, B_{i}, b_{i}\right) / A_{0}=f\left(\bar{\mu}_{1}, \bar{\lambda}_{2}, \bar{\mu}_{2}, \bar{\rho}_{2}, \bar{\gamma}_{2}, \bar{\alpha}_{2}, \bar{\beta}_{2}, \bar{\kappa}_{2}, \bar{j}_{2}, \bar{h}, \theta_{0}\right)
$$

where

$$
\begin{array}{ll}
\bar{\mu}_{1}=\frac{\mu_{1}}{\lambda_{1}}, & \bar{\lambda}_{2}=\frac{\lambda_{2}}{\lambda_{1}}, \quad \bar{\mu}_{2}=\frac{\mu_{2}}{\mu_{1}}, \quad \bar{\rho}_{2}=\frac{\rho_{2}}{\rho_{1}}, \quad \bar{\gamma}_{2}=\frac{\gamma_{2}}{\rho_{1} L_{0}^{4} \omega_{0}^{2}}, \\
\bar{\alpha}_{2}=\frac{\alpha_{2}}{\gamma_{2}}, & \bar{\beta}_{2}=\frac{\beta_{2}}{\gamma_{2}},
\end{array}
$$

In the present numerical examples, the main concerns are focused on the influences of the nondimensional micropolar constants $\bar{\alpha}_{2}, \bar{\beta}_{2}, \bar{\gamma}_{2}, \bar{\kappa}_{2}, \bar{j}_{2}$ and the nondimensional thickness $\bar{h}$ of the slab upon the reflection and transmission coefficients. The choice of these nondimensional micropolar constants in the numerical 

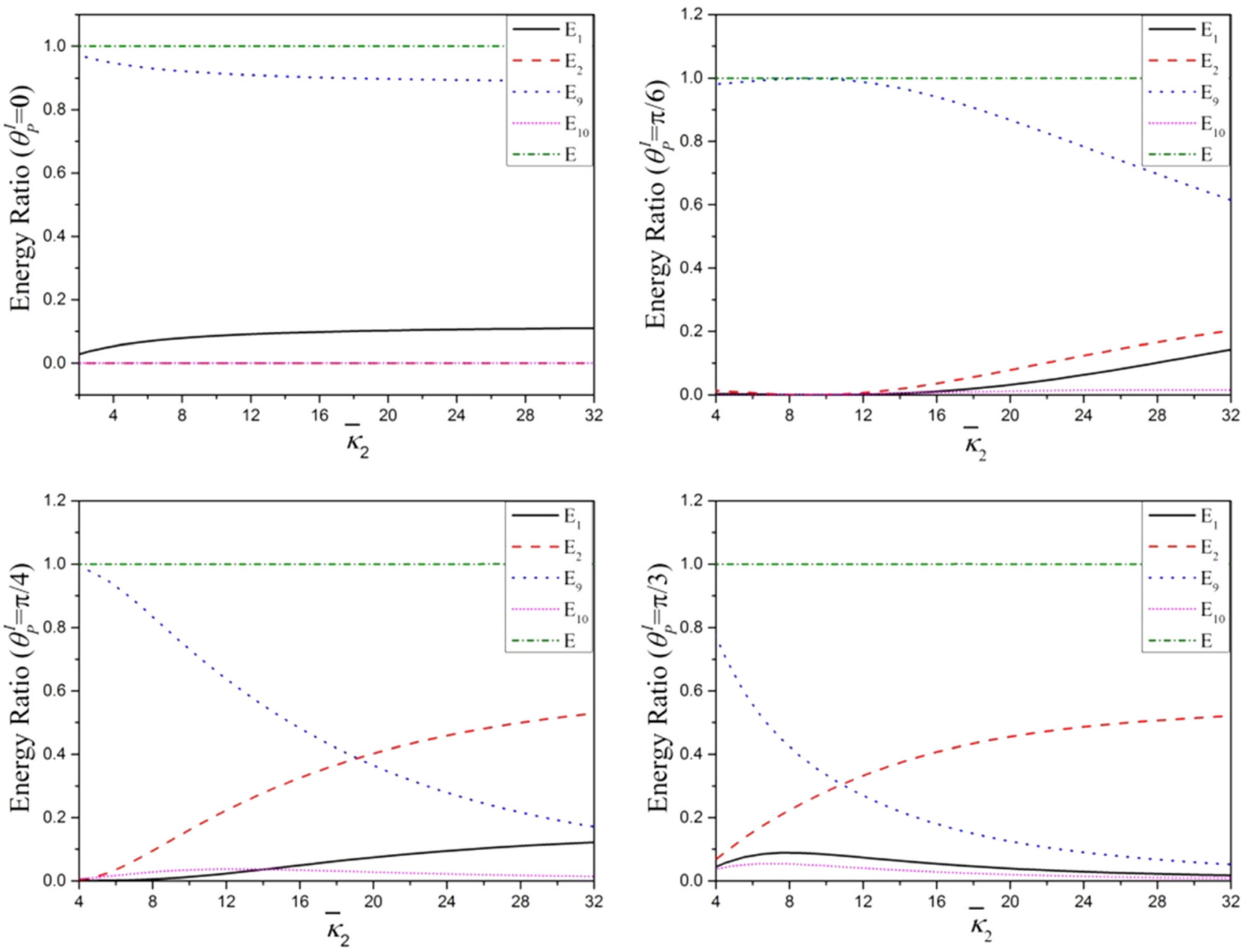

Fig. 9 The dependence of the reflection and transmission coefficients upon the micropolar constant $\bar{\kappa}_{2}$ for different incident angles $\theta_{P}^{\prime}$ in the case of incident $P$ wave $\left(d=0, \bar{\mu}_{1}=0.75, \bar{\lambda}_{2}=0.8, \bar{\mu}_{2}=0.6, \bar{\rho}_{2}=1.2, \bar{\gamma}_{2}=4, \bar{j}_{2}=30, \bar{h}=0.1\right)$

examples is not arbitrary completely but has taken into the range of micropolar constants of actual solid into consideration. The experiment results of micropolar constants of some actual solids are provided by Gauthier [20] and referenced in the present numerical example.

5.1 Incident $\boldsymbol{P}$ Wave Situation. Figure 3 shows the dependence of reflection and transmission coefficients on the nondimensional thickness $\bar{h}$. It is observed that the reflection and transmission $P$ waves are of periodicity. At certain frequencies, the reflection $P$ wave disappears nearly while the transmitted $P$ wave reaches maximum. This means that the elaborated sandwiched structure can be used as a frequency filter. Indeed, the sandwiched slab characteristic of classically elasticity can be used as a frequency filter too. However, the filter quality may be improved by the elaborate design of microstructure for the sandwiched slab characteristic of micropolar elasticity. The reflection and transmission $S V$ waves are approximately periodical but the energy carried by the reflection $S V$ waves is smaller by an order of magnitude than the reflection and transmission $P$ waves. The transmission $S V$ wave is smaller by two orders of magnitude than the reflection and transmission $P$ waves. In other words, the incident energy is mainly concentrated upon the reflection and transmission $P$ waves.

In order to make a comparison with that in literature [19], the dependences of energy ratios on the absolute thickness of the sandwiched layer are shown in Fig. 4. By comparing Fig. 4 in the present work with Fig. 3 in literature [19], not only energy ratios but also the fluctuating period exhibits a large deviation. It claims that the fluctuating period is a half of wavelength and the wavelength of $\mathrm{LD}$ wave is $2.58 \times 10^{-3} \mathrm{~m}$. However, after carefully analysis, it is found that the wavelength should be $16.20 \times$ $10^{-3} \mathrm{~m}\left(2 \pi \times 2.58 \times 10^{-3} \mathrm{~m}\right)$ at $0.5 \mathrm{MHz}$ for the material parameters provided in literature [19]. This mistake can thus explain the large deviations between our results and that in literature [19]. Unfortunately, the other figures in literature [19] also cannot be repeated and is thus doubtable. In a sense, the present work is motivated by providing the corrected numerical results for reference and comparison of related researches hereafter.

Figures 5 and 6 show the dependence of reflection and transmission coefficients on the micropolar constant $\bar{\gamma}_{2}$. It is observed that the micropolar constant $\bar{\gamma}_{2}$ has no any influence on the reflection and transmission coefficients in the normal incidence. In the oblique incidence, the micropolar constant $\bar{\gamma}_{2}$ has an evident influence on the reflection and transmission coefficients only at a range centered at $\bar{\gamma}_{2} \approx 3.0$. This range gradually increases as the incident angle increases. When the micropolar constant $\bar{\gamma}_{2}<3.0$, the reflection waves and the transmission $S V$ wave increase gradually as the micropolar constant $\bar{\gamma}_{2}$ increases while decrease gradually as the micropolar constant $\bar{\gamma}_{2}$ increases when $\bar{\gamma}_{2}>3$.0. It is also noted from Fig. 6 that the influence of the micropolar constant $\bar{\gamma}_{2}$ decreases gradually as the incident angle $\theta_{P}^{I}$ trends to zero and $\pi / 2$. The micropolar constants $\bar{\alpha}_{2}$ and $\bar{\beta}_{2}$ have similar influences as the micropolar constants $\bar{\gamma}_{2}$. Therefore, the figures about the micropolar constants $\bar{\alpha}_{2}$ and $\bar{\beta}_{2}$ are not given here for brevity. 

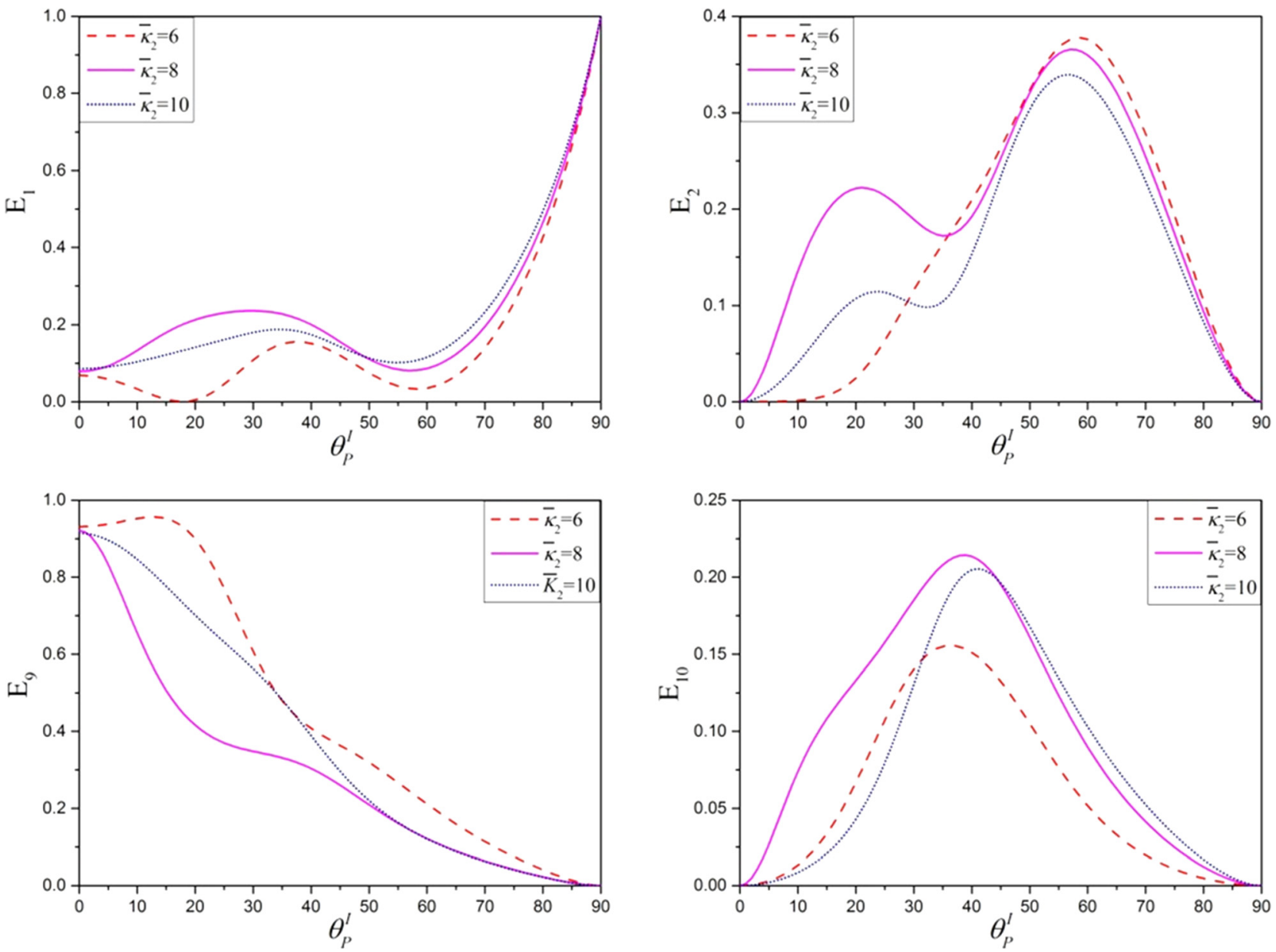

Fig. 10 The dependence of the reflection and transmission coefficients upon the incident angle $\theta_{P}^{I}$ for different micropolar constant $\bar{\kappa}_{2}$ in the case of incident $P$ wave $\left(d=0, \bar{\mu}_{1}=0.75, \bar{\lambda}_{2}=0.8, \bar{\mu}_{2}=0.6, \bar{\rho}_{2}=1.2, \bar{\gamma}_{2}=4, \bar{j}_{2}=30, \bar{h}=0.1\right)$
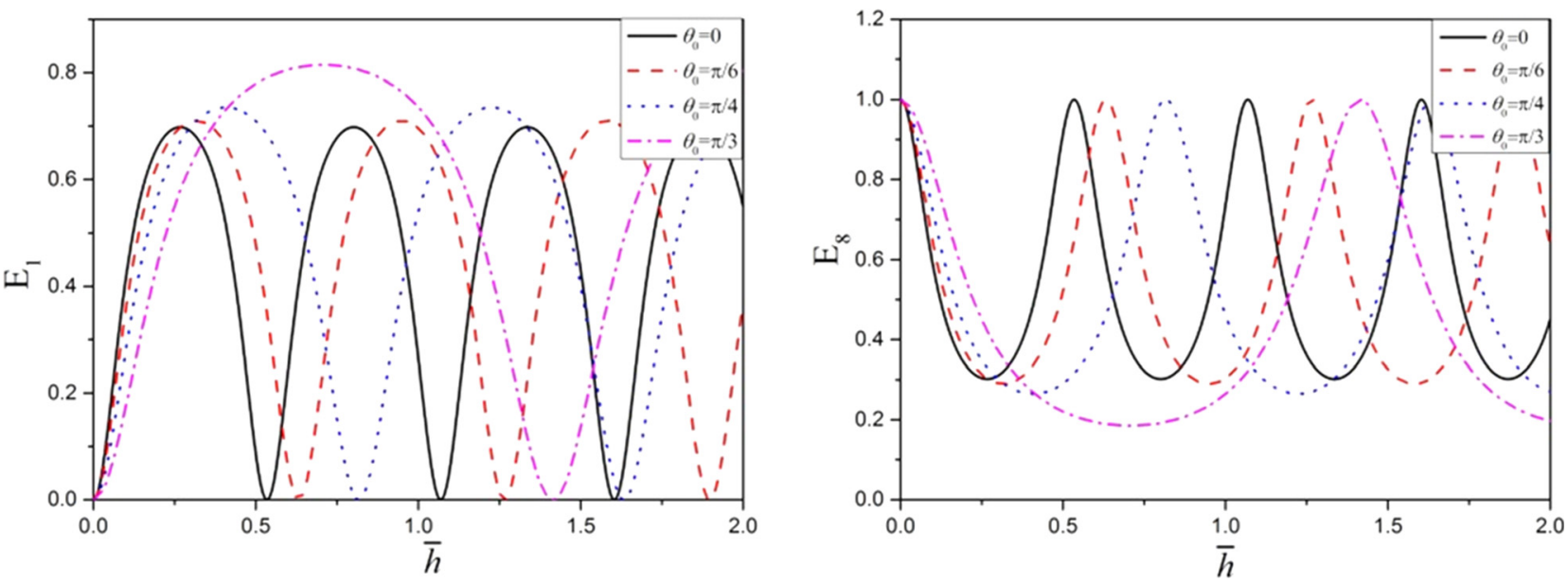

Fig. 11 The dependence of the reflection and transmission coefficients upon nondimensional thickness $\bar{h}$ in case of incident SH wave $\left(d=0, \bar{\mu}_{1}=0.2, \bar{\lambda}_{2}=0.2, \bar{\mu}_{2}=0.1, \bar{\rho}_{2}=0.7, \bar{\alpha}_{2}=\bar{\beta}_{2}=\bar{\gamma}_{2}=0.1, \bar{\kappa}_{2}=0.06, \bar{j}_{2}=1\right)$

Because the three micropolar constants are related with the couple stress, see Eq. ( $2 b)$, their influences reflect the influences of couple stress actually. Therefore, the phenomenon that their influences are extremely similar is understandable.

Figures 7 and 8 show the influences of the micro-inertia $\bar{j}_{2}$. The microrotation is a unique feature of the mass particle in the micropolar solid. The influences of the micro-inertia $\bar{j}_{2}$ actually reflect the influence of the microrotation. It is observed that there is a range centered at $\bar{j}_{2} \approx 38$ where the micro-inertia $\bar{j}_{2}$ has evident influences on the reflection and transmission waves. But the influences are only evident in the oblique incidence and decrease gradually when the incident angle tends to zero and $\pi / 2$. By 

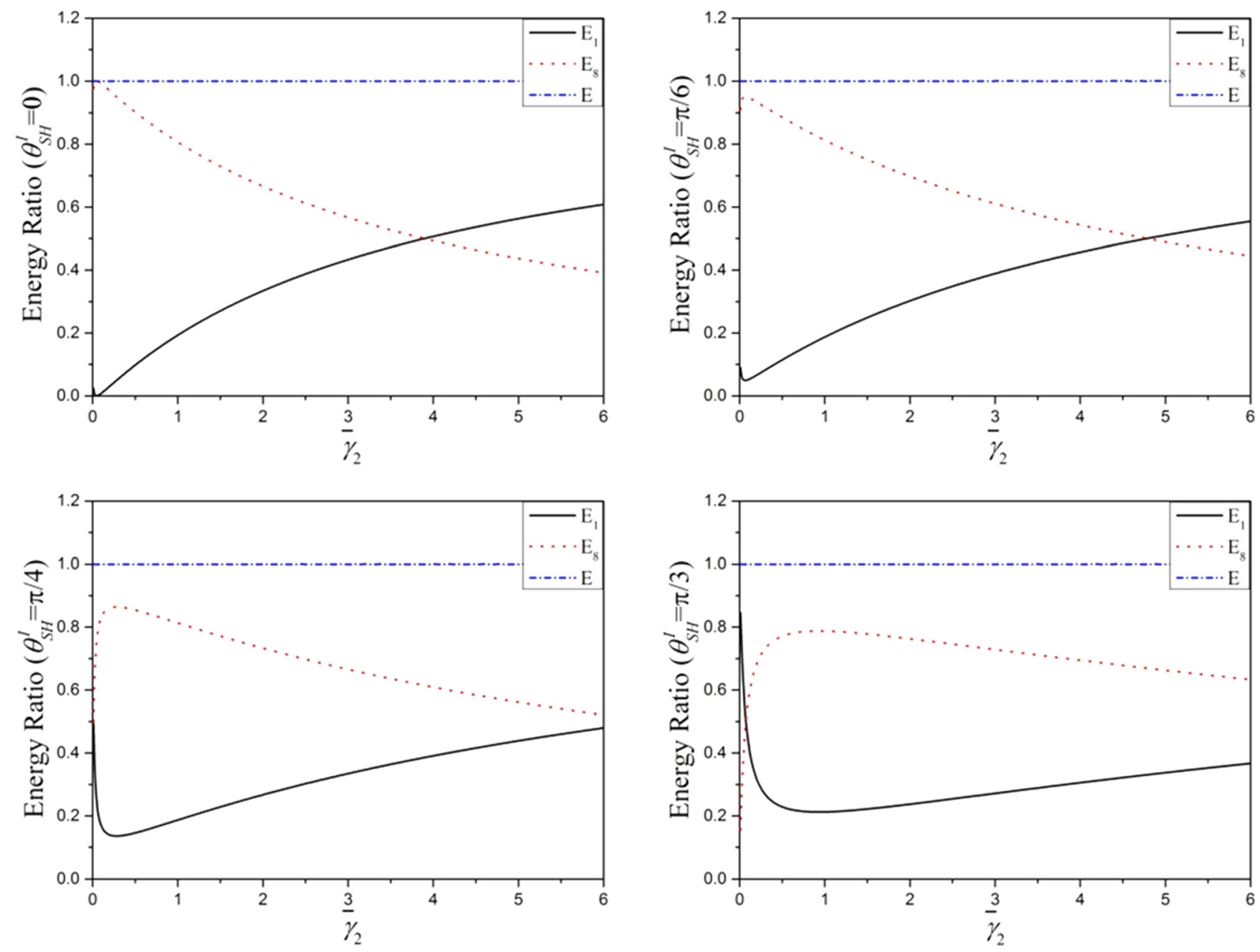

Fig. 12 The dependence of reflection and transmission coefficients upon the micropolar constant $\bar{\gamma}_{2}\left(\bar{\alpha}_{2}=\bar{\beta}_{2}=\bar{\gamma}_{2}\right)$ for different incident angles $\theta_{S H}^{\prime}$ in the case of incident $S H$ wave $\left(d=0, \bar{\mu}_{1}=0.2, \bar{\lambda}_{2}=0.2, \bar{\mu}_{2}=0.15, \bar{\rho}_{2}=0.7, \bar{\kappa}_{2}=1, \bar{j}_{2}=1, \bar{h}_{2}=0.1\right)$
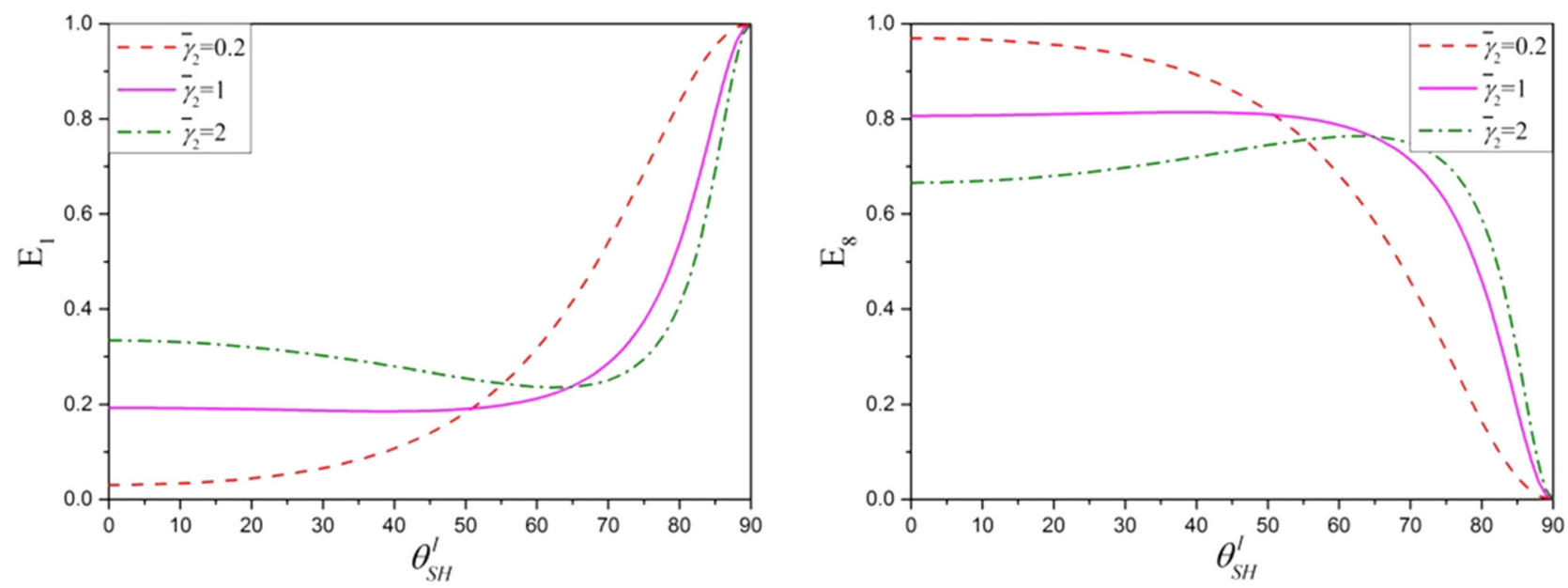

Fig. 13 The dependence of the reflection and transmission coefficients upon the incident angle $\theta_{S H}^{l}$ for different micropolar constants $\bar{\gamma}_{2}\left(\bar{\alpha}_{2}=\bar{\beta}_{2}=\bar{\gamma}_{2}\right)$ in the case of incident $S H$ wave $\left(d=0, \bar{\mu}_{1}=0.2, \bar{\lambda}_{2}=0.2, \bar{\mu}_{2}=0.15, \bar{\rho}_{2}=0.7, \bar{\kappa}_{2}=1, \bar{j}_{2}=1, \bar{h}_{2}=0.1\right)$

comparing with Figs. 5 and 7, it is found that the influences of the micro-inertia $\bar{j}_{2}$ are very similar with the influences of the micropolar constant $\bar{\gamma}_{2}$. This means that the influences of microrotation are consistent with the influences of couple stress.

Figures 9 and 10 show the influences of the micropolar constant $\bar{\kappa}_{2}$. It is noted from Eq. (1) that the micropolar constant $\bar{\kappa}_{2}$ plays a role of coupled coefficient which reflects the interaction between the displacement field and the microrotation field. Different from the micropolar constants $\bar{\alpha}_{2}, \bar{\beta}_{2}$, and $\bar{\gamma}_{2}$, there is not a finite influence range for the micropolar constant $\bar{\kappa}_{2}$. It is observed that the reflection coefficients increases monotonically while the transmission $P$ wave decreases monotonically as the micropolar constant $\bar{\kappa}_{2}$ increases gradually when the incident angle is less than $\pi / 4$. 

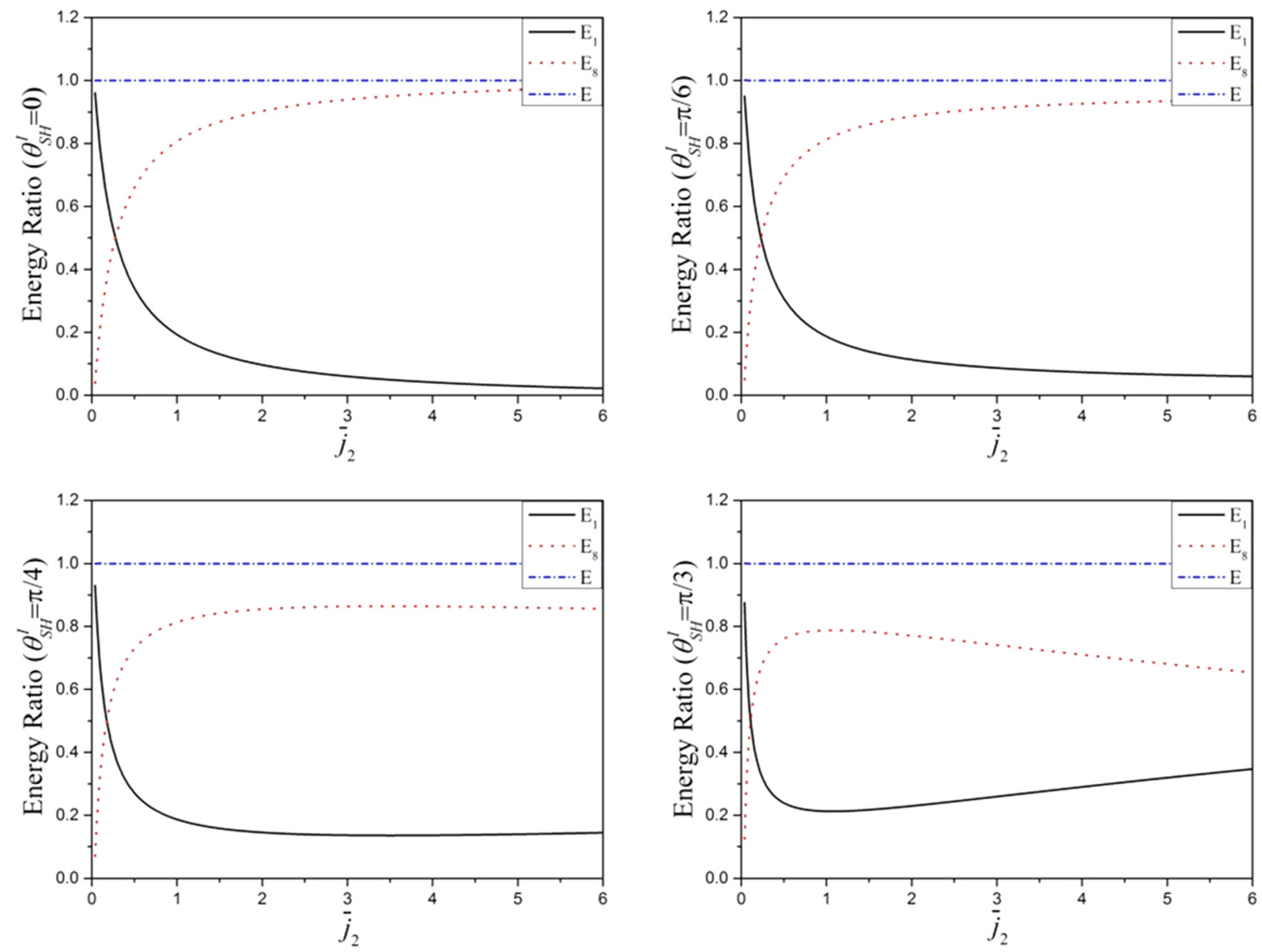

Fig. 14 The dependence of reflection and transmission coefficients upon the micro-inertia $\bar{j}_{2}$ for different incident angle $\theta_{S H}^{\prime}$ in the case of incident $S H$ wave $\left(d=0, \bar{\mu}_{1}=0.2, \bar{\lambda}_{2}=0.2, \bar{\mu}_{2}=0.15, \bar{\rho}_{2}=0.7, \bar{\alpha}_{2}=\bar{\beta}_{2}=\bar{\gamma}_{2}=1, \bar{\kappa}_{2}=1, \bar{h}_{2}=0.1\right)$
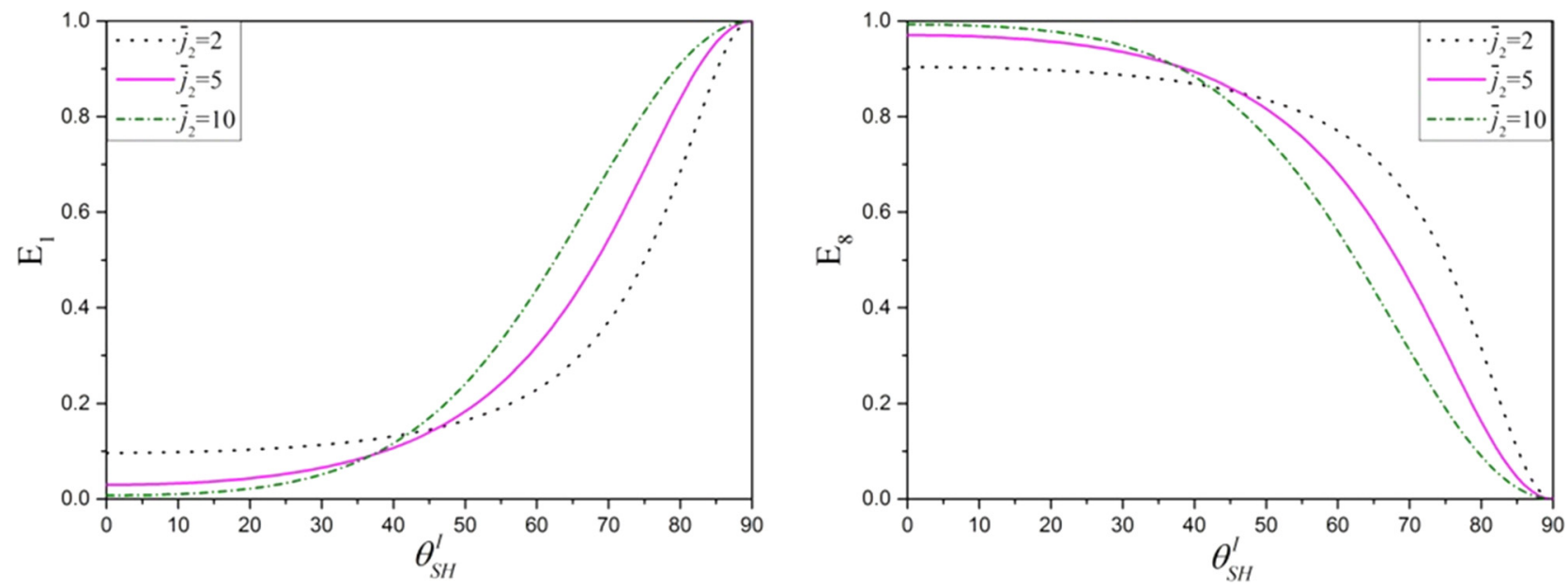

Fig. 15 The dependence of the reflection and transmission coefficients upon the incident angle $\theta_{S H}^{I}$ for different micro-inertia $\bar{j}_{2}$ in the case of incident $S H$ wave $\left(d=0, \bar{\mu}_{1}=0.2, \bar{\lambda}_{2}=0.2, \bar{\mu}_{2}=0.15, \bar{\rho}_{2}=0.7, \bar{\alpha}_{2}=\bar{\beta}_{2}=\bar{\gamma}_{2}=1, \bar{\kappa}_{2}=1, \bar{h} \bar{h}_{2}=0.1\right)$

In order to validate the numerical results, the energy conservation is checked for all numerical calculations. In Figs. 5, 7, and 9, the energy conservation index $E$ always keeps nearly equal to unit which signifies the energy conservation satisfied by the incident wave, the reflection waves and the transmission waves.

5.2 Incident $\boldsymbol{S H}$ Wave Situation. Figure 11 shows the dependence of reflection and transmission coefficients on the nondimensional thickness $\bar{h}$. It is observed that the reflection and transmission coefficients of $S H$ waves are periodically dependent upon the $\bar{h}$. At certain $\bar{h}$, the reflection $S H$ wave disappears and the total transmission phenomenon takes place. This signifies that the incident $S H$ wave can transmit the micropolar slab without any resistance. At other certain $\bar{h}$, the transmitted $S H$ wave reaches minimum but never equal to zero. This means that the total reflection phenomenon can never takes place. 

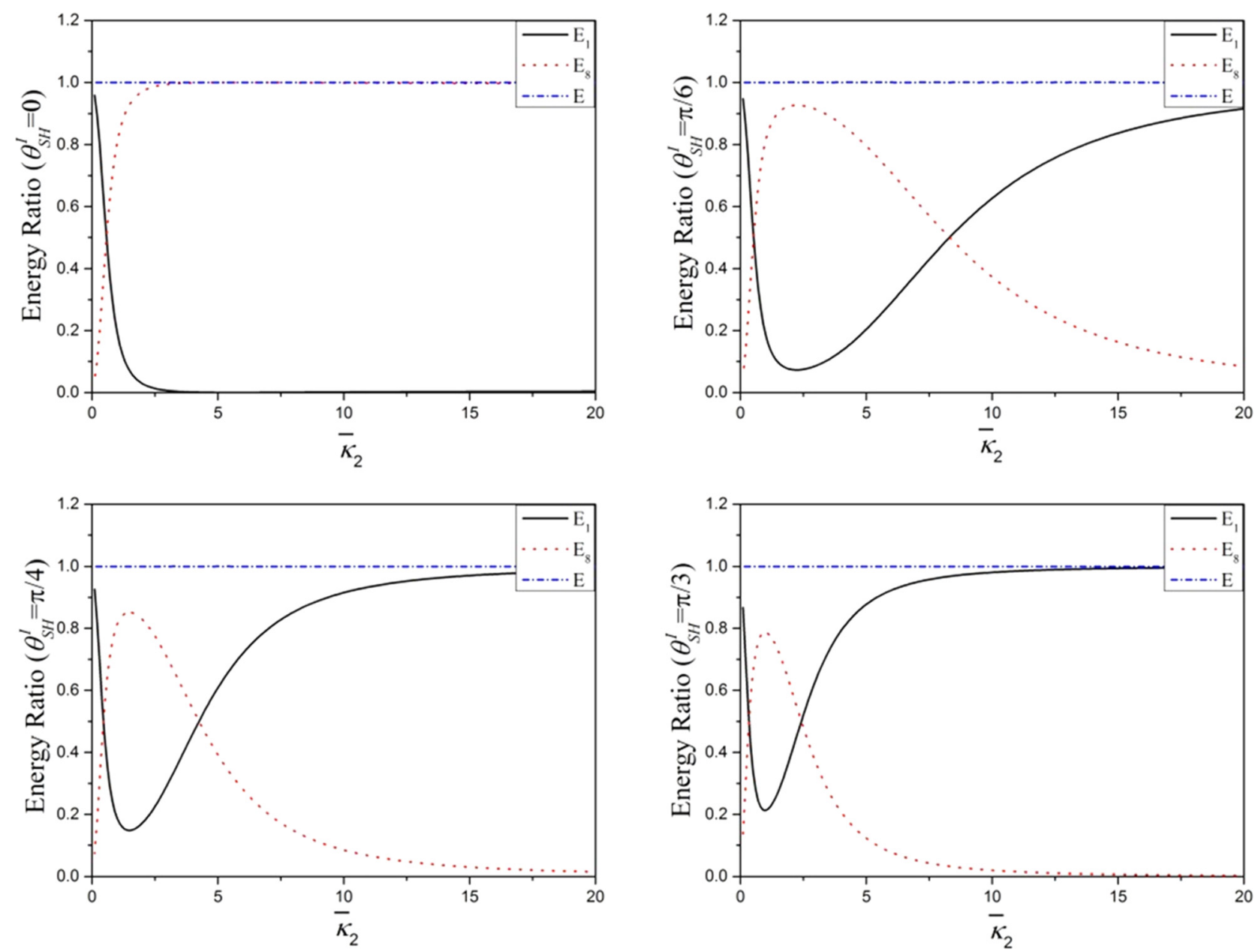

Fig. 16 The dependence of the reflection and transmission coefficients upon the micropolar constant $\bar{\kappa}_{2}$ for different incident angles $\theta_{S H}^{I}$ in the case of incident $S H$ wave $\left(d=0, \bar{\mu}_{1}=0.2, \bar{\lambda}_{2}=0.2, \bar{\mu}_{2}=0.15, \bar{\rho}_{2}=0.7, \bar{\alpha}_{2}=\bar{\beta}_{2}=\bar{\gamma}_{2}=1, \bar{j}_{2}=1, \bar{h}_{2}=0.1\right)$
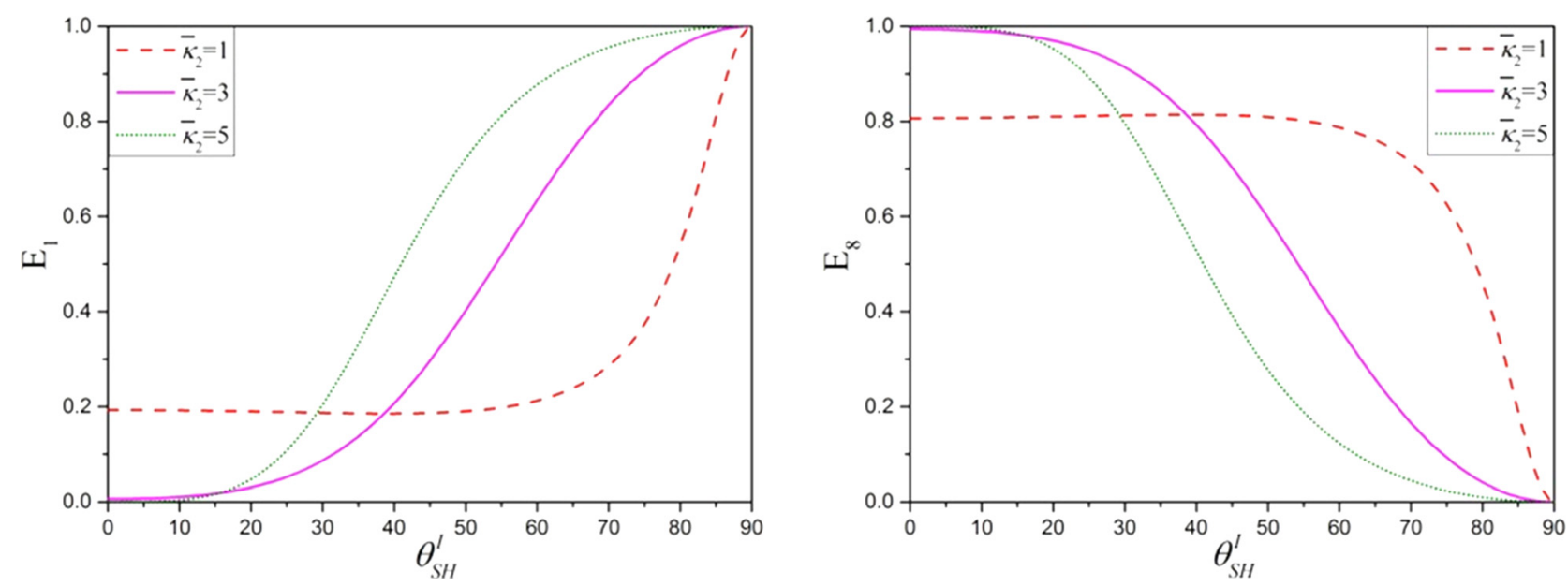

Fig. 17 The dependence of the reflection and transmission coefficients upon the incident angle $\theta_{S H}^{l}$ for different micropolar constants $\bar{\kappa}_{2}$ in the case of incident $S H$ wave $\left(d=0, \bar{\mu}_{1}=0.2, \bar{\lambda}_{2}=0.2, \bar{\mu}_{2}=0.15, \bar{\rho}_{2}=0.7, \bar{\alpha}_{2}=\bar{\beta}_{2}=\bar{\gamma}_{2}=1, \bar{j}_{2}=1, \bar{h}_{2}=0.1\right)$

Figures 12 and 13 show the dependence of reflection and transmission waves upon the micropolar constant $\bar{\gamma}_{2}$. Different from the incident $P$ wave, there is not a finite influence range for the micropolar constant $\bar{\gamma}_{2}$. It is observed that the reflection $S H$ wave increases monotonically while the transmission $S H$ wave decreases monotonically as the micropolar constant $\bar{\gamma}_{2}$ increases gradually when the incident angle is smaller than $\pi / 4$. When the incident angle is greater than $\pi / 4$, the reflection $S H$ wave decreases first then increases monotonically while the transmission $S H$ wave increases first then decreases monotonically. Different from the incident $P$ wave, the micropolar constant $\bar{\gamma}_{2}$ has still evident influences on the reflection and transmission waves even at normal incidence, see Fig. 13.

Figures 14 and 15 show the dependence of reflection and transmission waves upon the micro-inertia $\bar{j}_{2}$. Different from the incident $P$ wave, there is not a finite influence range for the 
micro-inertia $\bar{j}_{2}$. It is observed that the reflection $S H$ wave decreases monotonically while the transmission $S H$ wave increases monotonically as the micro-inertia $\bar{j}_{2}$ increases when the incident angle is smaller than $\pi / 4$.

Figures 16 and 17 show the dependence of the reflection and transmission $S H$ waves upon the micropolar constant $\bar{\kappa}_{2}$. It is observed that the reflection $S H$ wave decreases first then increases monotonically while the transmission $S H$ wave increases first then decreases monotonically as the micropolar constant $\bar{\kappa}_{2}$ the increases. This trend is different from the incident $P$ wave situation completely.

Similar with the case of incident $P$ wave, the energy conservation is checked in all numerical calculation in the case of incident $S H$ wave. The energy conservation index $E$ is shown in Figs. 12, 14 , and 16 . It is noted that the energy conservation index $E$ always keeps unit which means that the energy conservation is satisfied.

\section{Conclusions}

The reflection and transmission problem of an elastic wave through a micropolar slab sandwiched by two classic elastic halfspaces is studied in the present work. The interface conditions between the micropolar slab and the classic elastic half-spaces used in previous literature are modified in the present work as a generalized form. Based on the modified interface conditions, the reflection waves and the transmission waves are evaluated for the incident $P$ wave and the incident $S H$ wave. The influences of the micropolar constants and the thickness of slab are main concerns of the present work. From the numerical results, the following conclusions can be drawn:

(1) The reflection and the transmission coefficients are periodically dependent upon the thickness of slab. At certain thickness, the incident $P$ wave and the incident $S H$ wave can propagate through the slab without any resistance. Therefore, the sandwiched slab can be used as an acoustic frequency filter and the filter quality can be improved by the elaborated design of microstructure.

(2) Not only the micropolar constants $\bar{\alpha}_{2}, \bar{\beta}_{2}$, and $\bar{\gamma}_{2}$ have similar influences upon the reflection waves and the transmission waves but also only at a finite range, the reflection, and transmission waves are sensitive to the three micropolar constants. The three micropolar constants are all related with the couple stress, their influences reflect the influences of couple stress in the interface conditions actually.

(3) The influences of the micro-inertia $\bar{j}_{2}$ are similar with that the micropolar constants $\bar{\alpha}_{2}, \bar{\beta}_{2}$, and $\bar{\gamma}_{2}$. The micro-inertia $\bar{j}_{2}$ has also a finite range where the micro-inertia has evident influences on the reflection and transmission waves. The micro-inertia $\bar{j}_{2}$ is related with the microrotation and therefore reflects the influences of the microrotation in the interface conditions actually.

(4) The micropolar constant $\bar{\kappa}_{2}$ plays a role of coupled coefficient which reflects the interaction between the displacement field and the microrotation field. Different from the micropolar constants $\bar{\alpha}_{2}, \bar{\beta}_{2}, \bar{\gamma}_{2}$, and $\bar{j}_{2}$, there is not a finite influence range for the micropolar constant $\bar{\kappa}_{2}$. The influences of $\bar{\kappa}_{2}$ on the reflection and transmission waves are nearly monotonous.

(5) The waves in the micropolar slab can store and release energy periodically but does not absorb the energy. The incident energy is allocated between the reflection and transmission waves. In the incident $P$ wave situation, the incident energy concentrated mainly on the reflection and transmission $P$ waves while the reflection and transmission $S V$ waves are much weaker than the reflection and transmission $P$ waves.

\section{Acknowledgment}

The work was supported by Fundamental Research Funds for the Central Universities (FRF-BR-15-026A), National Natural
Science Foundation of China (No. 10972029), and Opening Fund of State Key Laboratory of Nonlinear Mechanics (LNM).

\section{Appendix A}

In the case of incident $P$ wave, the incident wave (indicated by superscript " $I$ "), the reflection waves (indicated by superscript " $R$ "), the transmission waves (indicated by superscript " $T$ ") in classical elastic media and the forward waves (indicated by superscript "(+)"), the backward waves (indicated by superscript "(-)") in micropolar slab can be written as follows:

$$
\begin{gathered}
q^{I}=a^{I} \exp \left[i k_{P}^{I}\left(\sin \theta_{P}^{I} x+\cos \theta_{P}^{I} z\right)-i \omega_{P}^{I} t\right] \\
q^{R}=a^{R} \exp \left[i k_{P}^{R}\left(\sin \theta_{P}^{R} x-\cos \theta_{P}^{R} z\right)-i \omega_{P}^{R} t\right] \\
U_{2}^{R}=A^{R} \exp \left[i k_{S V}^{R}\left(\sin \theta_{S V}^{R} x-\cos \theta_{S V}^{R} z\right)-i \omega_{S V}^{R} t\right] \\
q^{(+)}=a^{(+)} \exp \left[i k_{1}^{(+)}\left(\sin \theta_{1}^{(+)} x+\cos \theta_{1}^{(+)} z\right)-i \omega_{1}^{(+)} t\right] \\
U_{2}^{(+)}=\sum_{p=3,4} A_{p}^{(+)} \exp \left[i k_{p}^{(+)}\left(\sin \theta_{p}^{(+)} x+\cos \theta_{p}^{(+)} z\right)-i \omega_{p}^{(+)} t\right] \\
\varphi_{2}^{(+)}=\sum_{p=3,4} \eta_{p} A_{p}^{(+)} \exp \left[i k_{p}^{(+)}\left(\sin \theta_{p}^{(+)} x+\cos \theta_{p}^{(+)} z\right)-i \omega_{p}^{(+)} t\right] \\
q^{(-)}=a^{(-)} \exp \left[i k_{1}^{(-)}\left(\sin \theta_{1}^{(-)} x-\cos \theta_{1}^{(-)} z\right)-i \omega_{1}^{(-)} t\right] \\
U_{2}^{(-)}=\sum_{p=3,4} A_{p}^{(-)} \exp \left[i k_{p}^{(-)}\left(\sin \theta_{p}^{(-)} x-\cos \theta_{p}^{(-)} z\right)-i \omega_{p}^{(-)} t\right] \\
q_{2}^{T}=a^{T} \exp \left[i k_{P}^{T}\left(\sin \theta_{P}^{T} x+\cos \theta_{P}^{T} z\right)-i \omega_{P}^{T} t\right] \\
\varphi_{p=3,4} \eta_{p} A_{p}^{(-)} \exp \left[i k_{S V}^{T}\left(\sin \theta_{S V}^{T} x+\cos \theta_{S V}^{T} z\right)-i \omega_{S V}^{T} t\right]
\end{gathered}
$$

where $\omega_{i}=k_{i} v_{i}, p=3,4$ stand for two sets of CT wave, respectively. Derived from Eq. (7), the coupled coefficients $\eta_{3,4}$ are

$$
\eta_{3,4}=-\frac{\omega_{0}^{2}}{v_{3,4}^{2}-2 \omega_{0}^{2} / k_{3,4}^{2}-c_{4}^{2}}
$$

In the case of incident $S H$ wave, the incident wave, the reflection waves, the transmission waves in classical elastic media, and the forward waves and the backward waves in the micropolar slab can be written as follows:

$$
\begin{aligned}
u_{2}^{I} & =B^{I} \exp \left[i k_{S H}^{I}\left(\sin \theta_{S H}^{I} x+\cos \theta_{S H}^{I} z\right)-i \omega_{S H}^{I} t\right] \\
u_{2}^{R} & =B^{R} \exp \left[i k_{S H}^{R}\left(\sin \theta_{S H}^{R} x-\cos \theta_{S H}^{R} z\right)-i \omega_{S H}^{R} t\right] \\
\xi^{(+)} & =b^{(+)} \exp \left[i k_{2}^{(+)}\left(\sin \theta_{2}^{(+)} x+\cos \theta_{2}^{(+)} z\right)-i \omega_{2}^{(+)} t\right] \\
u_{2}^{(+)} & =\sum_{p=3,4} B_{p}^{(+)} \exp \left[i k_{p}^{(+)}\left(\sin \theta_{p}^{(+)} x+\cos \theta_{p}^{(+)} z\right)-i \omega_{p}^{(+)} t\right]
\end{aligned}
$$




$$
\begin{aligned}
\Phi_{2}^{(+)} & =\sum_{p=3,4} \Gamma_{p} B_{p}^{(+)} \exp \left[i k_{p}^{(+)}\left(\sin \theta_{p}^{(+)} x+\cos \theta_{p}^{(+)} z\right)-i \omega_{p}^{(+)} t\right] \\
\xi^{(-)} & =b^{(-)} \exp \left[i k_{2}^{(-)}\left(\sin \theta_{2}^{(-)} x-\cos \theta_{2}^{(-)} z\right)-i \omega_{2}^{(-)} t\right] \\
u_{2}^{(-)} & =\sum_{p=3,4} B_{p}^{(-)} \exp \left[i k_{p}^{(-)}\left(\sin \theta_{p}^{(-)} x-\cos \theta_{p}^{(-)} z\right)-i \omega_{p}^{(-)} t\right] \\
\Phi_{2}^{(-)} & =\sum_{p=3,4} \Gamma_{p} B_{p}^{(-)} \exp \left[i k_{p}^{(-)}\left(\sin \theta_{p}^{(-)} x-\cos \theta_{p}^{(-)} z\right)-i \omega_{p}^{(-)} t\right]
\end{aligned}
$$

$$
u_{2}^{T}=B^{T} \exp \left[i k_{S H}^{T}\left(\sin \theta_{S H}^{T} x+\cos \theta_{S H}^{T} z\right)-i \omega_{S H}^{T} t\right]
$$

where $\omega_{i}=k_{i} v_{i}, p=3,4$ stand for two sets of CT wave, respectively. Derived from Eq. (7), the coupled coefficients $\Gamma_{3,4}$ are

$$
\Gamma_{3,4}=-\frac{\omega_{0}^{2}}{\omega_{3,4}^{2}-2 \omega_{0}^{2}-c_{4}^{2} k_{3,4}^{2}}
$$

\section{Appendix B}

In the case of incident $P$ wave, the explicit expressions of matrix $\mathbf{A}_{P}$ are

$$
\begin{aligned}
& a_{1,1}=k_{P}^{R} \sin \theta_{P}^{R}, \quad a_{1,2}=k_{S V}^{R} \cos \theta_{S V}^{R}, \quad a_{1,3}=-k_{1}^{(+)} \sin \theta_{1}^{(+)}, \quad a_{1,4}=k_{3}^{(+)} \cos \theta_{3}^{(+)}, \quad a_{1,5}=k_{4}^{(+)} \cos \theta_{4}^{(+)}, \\
& a_{1,6}=-k_{1}^{(-)} \sin \theta_{1}^{(-)}, \quad a_{1,7}=-k_{3}^{(-)} \cos \theta_{3}^{(-)}, \quad a_{1,8}=-k_{4}^{(-)} \cos \theta_{4}^{(-)}, \quad a_{1,9}=0, \quad a_{1,10}=0, \\
& a_{2,1}=-k_{P}^{R} \cos \theta_{P}^{R}, \quad a_{2,2}=k_{S V}^{R} \sin \theta_{S V}^{R}, \quad a_{2,3}=-k_{1}^{(+)} \cos \theta_{1}^{(+)}, \quad a_{2,4}=-k_{3}^{(+)} \sin \theta_{3}^{(+)}, \\
& a_{2,5}=-k_{4}^{(+)} \sin \theta_{4}^{(+)}, \quad a_{2,6}=k_{1}^{(-)} \cos \theta_{1}^{(-)}, \quad a_{2,7}=-k_{3}^{(-)} \sin \theta_{3}^{(-)}, \quad a_{2,8}=-k_{4}^{(-)} \sin \theta_{4}^{(-)}, \quad a_{2,9}=0, \quad a_{2,10}=0, \\
& a_{3,1}=-\left(\lambda_{1}+2 \mu_{1} \cos ^{2} \theta_{P}^{R}\right)\left(k_{P}^{R}\right)^{2}, \quad a_{3,2}=2 \mu_{1} \sin \theta_{S V}^{R} \cos \theta_{S V}^{R}\left(k_{S V}^{R}\right)^{2}, \quad a_{3,3}=\left[\lambda_{2}+\left(2 \mu_{2}+\kappa_{2}\right) \cos ^{2} \theta_{1}^{(+)}\right]\left(k_{1}^{(+)}\right)^{2}, \\
& a_{3,4}=\left(2 \mu_{2}+\kappa_{2}\right) \sin \theta_{3}^{(+)} \cos \theta_{3}^{(+)}\left(k_{3}^{(+)}\right)^{2}, \quad a_{3,5}=\left(2 \mu_{2}+\kappa_{2}\right) \sin \theta_{4}^{(+)} \cos \theta_{4}^{(+)}\left(k_{4}^{(+)}\right)^{2}, \quad a_{3,6}=\left[\lambda_{2}+\left(2 \mu_{2}+\kappa_{2}\right) \cos ^{2} \theta_{1}^{(-)}\right]\left(k_{1}^{(-)}\right)^{2}, \\
& a_{3,7}=-\left(2 \mu_{2}+\kappa_{2}\right) \sin \theta_{3}^{(-)} \cos \theta_{3}^{(-)}\left(k_{3}^{(-)}\right)^{2}, \quad a_{3,8}=-\left(2 \mu_{2}+\kappa_{2}\right) \sin \theta_{4}^{(-)} \cos \theta_{4}^{(-)}\left(k_{4}^{(-)}\right)^{2}, \quad a_{3,9}=0, \\
& a_{3,10}=0, \quad a_{4,1}=2 \mu_{1} \sin \theta_{P}^{R} \cos \theta_{P}^{R}\left(k_{P}^{R}\right)^{2}, \quad a_{4,2}=\mu_{1} \cos 2 \theta_{S V}^{R}\left(k_{S V}^{R}\right)^{2}, \quad a_{4,3}=\left(2 \mu_{2}+\kappa_{2}\right) \sin \theta_{1}^{(+)} \cos \theta_{1}^{(+)}\left(k_{1}^{(+)}\right)^{2}, \\
& a_{4,4}=-\left(\mu_{2} \cos 2 \theta_{3}^{(+)}+\kappa_{2} \cos ^{2} \theta_{3}^{(+)}\right)\left(k_{3}^{(+)}\right)^{2}+\kappa_{2} \eta_{3}, \quad a_{4,5}=-\left(\mu_{2} \cos 2 \theta_{4}^{(+)}+\kappa_{2} \cos ^{2} \theta_{4}^{(+)}\right)\left(k_{4}^{(+)}\right)^{2}+\kappa_{2} \eta_{4}, \\
& a_{4,6}=\left(2 \mu_{2}+\kappa_{2}\right) \sin \theta_{1}^{(-)} \cos \theta_{1}^{(-)}\left(k_{1}^{(-)}\right)^{2}, \quad a_{4,7}=-\left(\mu_{2} \cos 2 \theta_{3}^{(-)}+\kappa_{2} \cos ^{2} \theta_{3}^{(-)}\right)\left(k_{3}^{(-)}\right)^{2}+\kappa_{2} \eta_{3}, \\
& a_{4,8}=-\left(\mu_{2} \cos 2 \theta_{4}^{(-)}+\kappa_{2} \cos ^{2} \theta_{4}^{(-)}\right)\left(k_{4}^{(-)}\right)^{2}+\kappa_{2} \eta_{4}, \quad a_{4,9}=0, \quad a_{4,10}=0, \\
& a_{5,1}=0, \quad a_{5,2}=0, \quad a_{5,3}=0, \quad a_{5,4}=i d \gamma_{2} k_{3}^{(+)} \eta_{3} \cos \theta_{3}^{(+)}+(1-d) K_{y} \eta_{3}, \\
& a_{5,5}=i d \gamma_{2} k_{4}^{(+)} \eta_{4} \cos \theta_{4}^{(+)}+(1-d) K_{y} \eta_{4}, \quad a_{5,6}=0, \quad a_{5,7}=-i d \gamma_{2} k_{3}^{(-)} \eta_{3} \cos \theta_{3}^{(-)}+(1-d) K_{y} \eta_{3}, \\
& a_{5,8}=-i d \gamma_{2} k_{4}^{(-)} \eta_{4} \cos \theta_{4}^{(-)}+(1-d) K_{y} \eta_{4}, \quad a_{5,9}=0, \quad a_{5,10}=0, \\
& a_{6,1}=0, \quad a_{6,2}=0, \quad a_{6,3}=k_{1}^{(+)} \sin \theta_{1}^{(+)} \exp \left(i k_{1}^{(+)} \cos \theta_{1}^{(+)} h\right), \quad a_{6,4}=-k_{3}^{(+)} \cos \theta_{3}^{(+)} \exp \left(i k_{3}^{(+)} \cos \theta_{3}^{(+)} h\right), \\
& a_{6,5}=-k_{4}^{(+)} \cos \theta_{4}^{(+)} \exp \left(i k_{4}^{(+)} \cos \theta_{4}^{(+)} h\right), \quad a_{6,6}=k_{1}^{(-)} \sin \theta_{1}^{(-)} \exp \left(-i k_{1}^{(-)} \cos \theta_{1}^{(-)} h\right), \\
& a_{6,7}=k_{3}^{(-)} \cos \theta_{3}^{(-)} \exp \left(-i k_{3}^{(-)} \cos \theta_{3}^{(-)} h\right), \quad a_{6,8}=k_{4}^{(-)} \cos \theta_{4}^{(-)} \exp \left(-i k_{4}^{(-)} \cos \theta_{4}^{(-)} h\right), \quad a_{6,9}=-k_{P}^{T} \sin \theta_{P}^{T} \exp \left(i k_{P}^{T} \cos \theta_{P}^{T} h\right), \\
& a_{6,10}=k_{S V}^{T} \cos \theta_{S V}^{T} \exp \left(i k_{S V}^{T} \cos \theta_{S V}^{T} h\right), \quad a_{7,1}=0, \quad a_{7,2}=0, \quad a_{7,3}=k_{1}^{(+)} \cos \theta_{1}^{(+)} \exp \left(i k_{1}^{(+)} \cos \theta_{1}^{(+)} h\right), \\
& a_{7,4}=k_{3}^{(+)} \sin \theta_{3}^{(+)} \exp \left(i k_{3}^{(+)} \cos \theta_{3}^{(+)} h\right), \quad a_{7,5}=k_{4}^{(+)} \sin \theta_{4}^{(+)} \exp \left(i k_{4}^{(+)} \cos \theta_{4}^{(+)} h\right), \\
& a_{7,6}=-k_{1}^{(-)} \cos \theta_{1}^{(-)} \exp \left(-i k_{1}^{(-)} \cos \theta_{1}^{(-)} h\right), \quad a_{7,7}=k_{3}^{(-)} \sin \theta_{3}^{(-)} \exp \left(-i k_{3}^{(-)} \cos \theta_{3}^{(-)} h\right), \\
& a_{7,8}=k_{4}^{(-)} \sin \theta_{4}^{(-)} \exp \left(-i k_{4}^{(-)} \cos \theta_{4}^{(-)} h\right), \quad a_{7,9}=-k_{P}^{T} \cos \theta_{P}^{T} \exp \left(i k_{P}^{T} \cos \theta_{P}^{T} h\right), \\
& a_{7,10}=-k_{S V}^{T} \cos \theta_{S V}^{T} \exp \left(i k_{S V}^{T} \cos \theta_{S V}^{T} h\right), \quad a_{8,1}=0, \quad a_{8,2}=0, \\
& a_{8,3}=-\left[\lambda_{2}+\left(2 \mu_{2}+\kappa_{2}\right) \cos ^{2} \theta_{1}^{(+)}\right]\left(k_{1}^{(+)}\right)^{2} \exp \left(i k_{1}^{(+)} \cos \theta_{1}^{(+)} h\right), \\
& a_{8,4}=-\left(2 \mu_{2}+\kappa_{2}\right) \sin \theta_{3}^{(+)} \cos \theta_{3}^{(+)}\left(k_{3}^{(+)}\right)^{2} \exp \left(i k_{3}^{(+)} \cos \theta_{3}^{(+)} h\right) \text {, } \\
& a_{8,5}=-\left(2 \mu_{2}+\kappa_{2}\right) \sin \theta_{4}^{(+)} \cos \theta_{4}^{(+)}\left(k_{4}^{(+)}\right)^{2} \exp \left(i k_{4}^{(+)} \cos \theta_{4}^{(+)} h\right) \text {, } \\
& a_{8,6}=-\left[\lambda_{2}+\left(2 \mu_{2}+\kappa_{2}\right) \cos ^{2} \theta_{1}^{(-)}\right]\left(k_{1}^{(-)}\right)^{2} \exp \left(-i k_{1}^{(-)} \cos \theta_{1}^{(-)} h\right) \text {, } \\
& a_{8,7}=\left(2 \mu_{2}+\kappa_{2}\right) \sin \theta_{3}^{(-)} \cos \theta_{3}^{(-)}\left(k_{3}^{(-)}\right)^{2} \exp \left(-i k_{3}^{(-)} \cos \theta_{3}^{(-)} h\right) \text {, } \\
& a_{8,8}=\left(2 \mu_{2}+\kappa_{2}\right) \sin \theta_{4}^{(-)} \cos \theta_{4}^{(-)}\left(k_{4}^{(-)}\right)^{2} \exp \left(i k_{4}^{(-)} \cos \theta_{4}^{(-)} h\right) \text {, } \\
& a_{8,9}=\left(\lambda_{1}+2 \mu_{1} \cos ^{2} \theta_{P}^{T}\right)\left(k_{P}^{T}\right)^{2} \exp \left(i k_{P}^{T} \cos \theta_{P}^{T} h\right), \quad a_{8,10}=2 \mu_{1} \sin \theta_{S V}^{T} \cos \theta_{S V}^{T}\left(k_{S V}^{T}\right)^{2} \exp \left(i k_{S V}^{T} \cos \theta_{S V}^{T} h\right), \\
& a_{9,1}=0, \quad a_{9,2}=0, \quad a_{9,3}=-\left(2 \mu_{2}+\kappa_{2}\right) \sin \theta_{1}^{(+)} \cos \theta_{1}^{(+)}\left(k_{1}^{(+)}\right)^{2} \exp \left(i k_{1}^{(+)} \cos \theta_{1}^{(+)} h\right),
\end{aligned}
$$




$$
\begin{aligned}
& a_{9,4}=\left[\left(\mu_{2} \cos 2 \theta_{3}^{(+)}+\kappa_{2} \cos ^{2} \theta_{3}^{(+)}\right)\left(k_{3}^{(+)}\right)^{2}-\kappa_{2} \eta_{3}\right] \exp \left(i k_{3}^{(+)} \cos \theta_{3}^{(+)} h\right), \\
& a_{9,5}= {\left[\left(\mu_{2} \cos 2 \theta_{4}^{(+)}+\kappa_{2} \cos ^{2} \theta_{4}^{(+)}\right)\left(k_{4}^{(+)}\right)^{2}-\kappa_{2} \eta_{4}\right] \exp \left(i k_{4}^{(+)} \cos \theta_{4}^{(+)} h\right), } \\
& a_{9,6}=\left(2 \mu_{2}+\kappa_{2}\right) \sin \theta_{1}^{(-)} \cos \theta_{1}^{(-)}\left(k_{1}^{(-)}\right)^{2} \exp \left(-i k_{1}^{(-)} \cos \theta_{1}^{(-)} h\right), \\
& a_{9,7}=\left[\left(\mu_{2} \cos 2 \theta_{3}^{(-)}+\kappa_{2} \cos ^{2} \theta_{3}^{(-)}\right)\left(k_{3}^{(-)}\right)^{2}-\kappa_{2} \eta_{3}\right] \exp \left(-i k_{3}^{(-)} \cos \theta_{3}^{(-)} h\right), \\
& a_{9,8}=\left[\left(\mu_{2} \cos 2 \theta_{4}^{(-)}+\kappa_{2} \cos ^{2} \theta_{4}^{(-)}\right)\left(k_{4}^{(-)}\right)^{2}-\kappa_{2} \eta_{4}\right] \exp \left(-i k_{4}^{(-)} \cos \theta_{4}^{(-)} h\right), \\
& a_{9,9}=2 \mu_{1} \sin \theta_{P}^{T} \cos \theta_{P}^{T}\left(k_{P}^{T}\right)^{2} \exp \left(i k_{P}^{T} \cos \theta_{P}^{T} h\right), \\
& a_{9,10}=-\mu_{1} \cos 2 \theta_{S V}^{T}\left(k_{S V}^{T}\right)^{2} \exp \left(i k_{S V}^{T} \cos \theta_{S V}^{T} h\right), \quad a_{10,1}=0, \quad a_{10,2}=0, \\
& a_{10,3}=0, \quad a_{10,4}=\left[i d \gamma_{2} k_{3}^{(+)} \eta_{3} \cos \theta_{3}^{(+)}+(1-d) K_{y} \eta_{3}\right] \exp \left(i k_{3}^{(+)} \cos \theta_{3}^{(+)} h\right), \\
& a_{10,5}=\left[i d \gamma_{2} k_{4}^{(+)} \eta_{4} \cos \theta_{4}^{(+)}+(1-d) K_{y} \eta_{4}\right] \exp \left(i k_{4}^{(+)} \cos \theta_{4}^{(+)} h\right), \quad a_{10,6}=0, \\
& a_{10,7}=\left[-i d \gamma_{2} k_{3}^{(-)} \eta_{3} \cos \theta_{3}^{(-)}+(1-d) K_{y} \eta_{3}\right] \exp \left(-i k_{3}^{(-)} \cos \theta_{3}^{(-)} h\right), \\
& a_{10,8}=\left[-i d \gamma_{2} k_{4}^{(-)} \eta_{4} \cos \theta_{4}^{(-)}+(1-d) K_{y} \eta_{4}\right] \exp \left(-i k_{4}^{(-)} \cos \theta_{4}^{(-)} h\right), \quad a_{10,9}=0, \quad a_{10,10}=0
\end{aligned}
$$

The explicit expressions of matrix $\mathbf{B}_{P}$ are

$$
b_{1}=-k_{P}^{I} \sin \theta_{P}^{I}, \quad b_{2}=-k_{P}^{I} \cos \theta_{P}^{I}, \quad b_{3}=\left(\lambda_{1}+2 \mu_{1} \cos ^{2} \theta_{P}^{I}\right)\left(k_{P}^{I}\right)^{2}, \quad b_{4}=2 \mu_{1} \sin \theta_{P}^{I} \cos \theta_{P}^{I}\left(k_{P}^{I}\right)^{2}, \quad b_{5}=b_{6}=b_{7}=b_{8}=b_{9}=b_{10}=0
$$

In the case of incident $S H$ wave, the explicit expressions of matrix $\mathbf{A}_{S}$ are

$$
\begin{aligned}
& a_{1,1}=-1, \quad a_{1,2}=0, \quad a_{1,3}=1, \quad a_{1,4}=1, \quad a_{1,5}=0, \quad a_{1,6}=1, \quad a_{1,7}=1, \quad a_{1,8}=0, \quad a_{2,1}=\mu_{1} k_{S H}^{R} \cos \theta_{S H}^{R}, \\
& a_{2,2}=\kappa_{2} k_{2}^{(+)} \sin \theta_{2}^{(+)}, \quad a_{2,3}=\left(\mu_{2}+\kappa_{2}-\kappa_{2} \Gamma_{3}\right) k_{3}^{(+)} \cos \theta_{3}^{(+)}, \quad a_{2,4}=\left(\mu_{2}+\kappa_{2}-\kappa_{2} \Gamma_{4}\right) k_{4}^{(+)} \cos \theta_{4}^{(+)}, \\
& a_{2,5}=\kappa_{2} k_{2}^{(-)} \sin \theta_{2}^{(-)}, \quad a_{2,6}=-\left(\mu_{2}+\kappa_{2}-\kappa_{2} \Gamma_{3}\right) k_{3}^{(-)} \cos \theta_{3}^{(-)}, \quad a_{2,7}=-\left(\mu_{2}+\kappa_{2}-\kappa_{2} \Gamma_{4}\right) k_{4}^{(-)} \cos \theta_{4}^{(-)} \text {, } \\
& a_{2,8}=0, \quad a_{3,1}=0, \quad a_{3,2}=-d(\beta+\gamma)\left(k_{2}^{(+)}\right)^{2} \sin \theta_{2}^{(+)} \cos \theta_{2}^{(+)}+i(1-d) K_{x} k_{2}^{(+)} \sin \theta_{2}^{(+)}, \\
& a_{3,3}=d \Gamma_{3}\left(k_{3}^{(+)}\right)^{2}\left(\gamma \cos ^{2} \theta_{3}^{(+)}-\beta \sin ^{2} \theta_{3}^{(+)}\right)-i(1-d) K_{x} \Gamma_{3} k_{3}^{(+)} \cos \theta_{3}^{(+)}, \\
& a_{3,4}=d \Gamma_{4}\left(k_{4}^{(+)}\right)^{2}\left(\gamma \cos ^{2} \theta_{4}^{(+)}-\beta \sin ^{2} \theta_{4}^{(+)}\right)-i(1-d) K_{x} \Gamma_{4} k_{4}^{(+)} \cos \theta_{4}^{(+)}, \\
& a_{3,5}=d(\beta+\gamma)\left(k_{2}^{(-)}\right)^{2} \sin \theta_{2}^{(-)} \cos \theta_{2}^{(-)}+i(1-d) K_{x} k_{2}^{(-)} \sin \theta_{2}^{(-)}, \\
& a_{3,6}=d \Gamma_{3}\left(k_{3}^{(-)}\right)^{2}\left(\gamma \cos ^{2} \theta_{3}^{(-)}-\beta \sin ^{2} \theta_{3}^{(-)}\right)+i(1-d) K_{x} \Gamma_{3} k_{3}^{(-)} \cos \theta_{3}^{(-)}, \\
& a_{3,7}=d \Gamma_{4}\left(k_{4}^{(-)}\right)^{2}\left(\gamma \cos ^{2} \theta_{4}^{(-)}-\beta \sin ^{2} \theta_{4}^{(-)}\right)+i(1-d) K_{x} \Gamma_{4} k_{4}^{(-)} \cos \theta_{4}^{(-)} \text {, } \\
& a_{3,8}=0, \quad a_{4,1}=0, \quad a_{4,2}=-d\left[\alpha+(\beta+\gamma) \cos ^{2} \theta_{2}^{(+)}\right]\left(k_{2}^{(+)}\right)^{2}+i(1-d) K_{z} k_{2}^{(+)} \cos \theta_{2}^{(+)}, \\
& a_{4,3}=-d(\beta+\gamma) \Gamma_{3}\left(k_{3}^{(+)}\right)^{2} \sin \theta_{3}^{(+)} \cos \theta_{3}^{(+)}+i(1-d) K_{z} \Gamma_{3} k_{3}^{(+)} \sin \theta_{3}^{(+)}, \\
& a_{4,4}=-d(\beta+\gamma) \Gamma_{4}\left(k_{4}^{(+)}\right)^{2} \sin \theta_{4}^{(+)} \cos \theta_{4}^{(+)}+i(1-d) K_{z} \Gamma_{4} k_{4}^{(+)} \sin \theta_{4}^{(+)} \text {, } \\
& a_{4,5}=-d\left[\alpha+(\beta+\gamma) \cos ^{2} \theta_{2}^{(-)}\right]\left(k_{2}^{(-)}\right)^{2}-i(1-d) K_{z} k_{2}^{(-)} \cos \theta_{2}^{(-)} \text {, } \\
& a_{4,6}=d(\beta+\gamma) \Gamma_{3}\left(k_{3}^{(-)}\right)^{2} \sin \theta_{3}^{(-)} \cos \theta_{3}^{(-)}+i(1-d) K_{z} \Gamma_{3} k_{3}^{(-)} \sin \theta_{3}^{(-)}, \\
& a_{4,7}=d(\beta+\gamma) \Gamma_{4}\left(k_{4}^{(-)}\right)^{2} \sin \theta_{4}^{(-)} \cos \theta_{4}^{(-)}+i(1-d) K_{z} \Gamma_{4} k_{4}^{(-)} \sin \theta_{4}^{(-)}, \\
& a_{4,8}=0, \quad a_{5,1}=0, \quad a_{5,2}=0, \quad a_{5,3}=\exp \left(i k_{3}^{(+)} \cos \theta_{3}^{(+)} h\right), \quad a_{5,4}=\exp \left(i k_{4}^{(+)} \cos \theta_{4}^{(+)} h\right), \\
& a_{5,5}=0, \quad a_{5,6}=\exp \left(-i k_{3}^{(-)} \cos \theta_{3}^{(-)} h\right), \quad a_{5,7}=\exp \left(-i k_{4}^{(-)} \cos \theta_{4}^{(-)} h\right), \quad a_{5,8}=-\exp \left(i k_{S H}^{T} \cos \theta_{S H}^{T} h\right), \\
& a_{6,1}=0, \quad a_{6,2}=\kappa_{2} k_{2}^{(+)} \sin \theta_{2}^{(+)} \exp \left(i k_{2}^{(+)} \cos \theta_{2}^{(+)} h\right), \quad a_{6,3}=\left(\mu_{2}+\kappa_{2}-\kappa_{2} \Gamma_{3}\right) k_{3}^{(+)} \cos \theta_{3}^{(+)} \exp \left(i k_{3}^{(+)} \cos \theta_{3}^{(+)} h\right), \\
& a_{6,4}=\left(\mu_{2}+\kappa_{2}-\kappa_{2} \Gamma_{4}\right) k_{4}^{(+)} \cos \theta_{4}^{(+)} \exp \left(i k_{4}^{(+)} \cos \theta_{4}^{(+)} h\right), \quad a_{6,5}=\kappa_{2} k_{2}^{(-)} \sin \theta_{2}^{(-)} \exp \left(-i k_{2}^{(-)} \cos \theta_{2}^{(-)} h\right), \\
& a_{6,6}=-\left(\mu_{2}+\kappa_{2}-\kappa_{2} \Gamma_{3}\right) k_{3}^{(-)} \cos \theta_{3}^{(-)} \exp \left(-i k_{3}^{(-)} \cos \theta_{3}^{(-)} h\right), \quad a_{6,7}=-\left(\mu_{2}+\kappa_{2}-\kappa_{2} \Gamma_{4}\right) k_{4}^{(-)} \cos \theta_{4}^{(-)} \exp \left(-i k_{4}^{(-)} \cos \theta_{4}^{(-)} h\right), \\
& a_{6,8}=-\mu_{1} k_{S H}^{T} \cos \theta_{S H}^{T} \exp \left(i k_{S H}^{T} \cos \theta_{S H}^{T} h\right), \quad a_{7,1}=0, \\
& a_{7,2}=\left[-d(\beta+\gamma) k_{2}^{2} \sin \theta_{2}^{(+)} \cos \theta_{2}^{(+)}+i(1-d) K_{x} k_{2} \sin \theta_{2}^{(+)}\right] \exp \left(i k_{2}^{(+)} \cos \theta_{2}^{(+)} h\right), \\
& a_{7,3}=\left[d \Gamma_{3}\left(k_{3}^{(+)}\right)^{2}\left(\gamma \cos ^{2} \theta_{3}^{(+)}-\beta \sin ^{2} \theta_{3}^{(+)}\right)-i(1-d) K_{x} \Gamma_{3} k_{3}^{(+)} \cos \theta_{3}^{(+)}\right] \exp \left(i k_{3}^{(+)} \cos \theta_{3}^{(+)} h\right),
\end{aligned}
$$




$$
\begin{aligned}
& a_{7,4}=\left[d \Gamma_{4}\left(k_{4}^{(+)}\right)^{2}\left(\gamma \cos ^{2} \theta_{4}^{(+)}-\beta \sin ^{2} \theta_{4}^{(+)}\right)-i(1-d) K_{x} \Gamma_{4} k_{4}^{(+)} \cos \theta_{4}^{(+)}\right] \exp \left(i k_{4}^{(+)} \cos \theta_{4}^{(+)} h\right), \\
& a_{7,5}=\left[d(\beta+\gamma)\left(k_{2}^{(-)}\right)^{2} \sin \theta_{2}^{(-)} \cos \theta_{2}^{(-)}+i(1-d) K_{x} k_{2}^{(-)} \sin \theta_{2}^{(-)}\right] \exp \left(-i k_{2}^{(-)} \cos \theta_{2}^{(-)} h\right), \\
& a_{7,6}=\left[d \Gamma_{3}\left(k_{3}^{(-)}\right)^{2}\left(\gamma \cos ^{2} \theta_{3}^{(-)}-\beta \sin ^{2} \theta_{3}^{(-)}\right)+i(1-d) K_{x} \Gamma_{3} k_{3}^{(-)} \cos \theta_{3}^{(-)}\right] \exp \left(-i k_{3}^{(-)} \cos \theta_{3}^{(-)} h\right), \\
& a_{7,7}=\left[d \Gamma_{4}\left(k_{4}^{(-)}\right)^{2}\left(\gamma \cos ^{2} \theta_{4}^{(-)}-\beta \sin ^{2} \theta_{4}^{(-)}\right)+i(1-d) K_{x} \Gamma_{4} k_{4}^{(-)} \cos \theta_{4}^{(-)}\right] \exp \left(-i k_{4}^{(-)} \cos \theta_{4}^{(-)} h\right), \\
& a_{7,8}=0, \quad a_{8,1}=0, \quad a_{8,2}=\left[-d\left[\alpha+(\beta+\gamma) \cos ^{2} \theta_{2}^{(+)}\right]\left(k_{2}^{(+)}\right)^{2}+i(1-d) K_{z} k_{2}^{(+)} \cos \theta_{2}^{(+)}\right] \exp \left(i k_{2}^{(+)} \cos \theta_{2}^{(+)} h\right), \\
& a_{8,3}=\left[-d(\beta+\gamma) \Gamma_{3}\left(k_{3}^{(+)}\right)^{2} \sin \theta_{3}^{(+)} \cos \theta_{3}^{(+)}+i(1-d) K_{z} \Gamma_{3} k_{3}^{(+)} \sin \theta_{3}^{(+)}\right] \exp \left(i k_{3}^{(+)} \cos \theta_{3}^{(+)} h\right), \\
& a_{8,4}=\left[-d(\beta+\gamma) \Gamma_{4}\left(k_{4}^{(+)}\right)^{2} \sin \theta_{4}^{(+)} \cos \theta_{4}^{(+)}+i(1-d) K_{z} \Gamma_{4} k_{4}^{(+)} \sin \theta_{4}^{(+)}\right] \exp \left(i k_{4}^{(+)} \cos \theta_{4}^{(+)} h\right), \\
& a_{8,5}=\left\{-d\left[\alpha+(\beta+\gamma) \cos ^{2} \theta_{2}^{(-)}\right]\left(k_{2}^{(-)}\right)^{2}-i(1-d) K_{z} k_{2}^{(-)} \cos \theta_{2}^{(-)}\right\} \exp \left(-i k_{2}^{(-)} \cos \theta_{2}^{(-)} h\right), \\
& a_{8,6}=\left[d(\beta+\gamma) \Gamma_{3}\left(k_{3}^{(-)}\right)^{2} \sin \theta_{3}^{(-)} \cos \theta_{3}^{(-)}+i(1-d) K_{z} \Gamma_{3} k_{3}^{(-)} \sin \theta_{3}^{(-)}\right] \exp \left(-i k_{3}^{(-)} \cos \theta_{3}^{(-)} h\right), \\
& a_{8,7}=\left[d(\beta+\gamma) \Gamma_{4}\left(k_{4}^{(-)}\right)^{2} \sin \theta_{4}^{(-)} \cos \theta_{4}^{(-)}+i(1-d) K_{z} \Gamma_{4} k_{4}^{(-)} \sin \theta_{4}^{(-)}\right] \exp \left(-i k_{4}^{(-)} \cos \theta_{4}^{(-)} h\right), \quad b_{8,8}=0
\end{aligned}
$$

The explicit expressions of matrix $\mathbf{B}_{S}$ are

$$
b_{1}=1, \quad b_{2}=\mu_{1} k_{S H}^{I} \cos \theta_{S H}^{I}, \quad b_{3}=b_{4}=b_{5}=b_{6}=b_{7}=b_{8}=0
$$

\section{References}

[1] Eringen, A. C., 2002, Nonlocal Continuum Field Theories, Springer Science \& Business Media, New York.

[2] Eringen, A. C., 1966, Mechanics of Micromorphic Materials, Springer, Berlin.

[3] Mindlin, R. D., and Tiersten, H. F., 1962, "Effects of Couple-Stresses in Linear Elasticity," Arch. Ration. Mech. Anal., 11(1), pp. 415-448.

[4] Toupin, R. A., 1964, "Theories of Elasticity With Couple-Stress," Arch. Ration. Mech. Anal., 17(2), pp. 85-112.

[5] Eringen, A. C., 1966, "Linear Theory of Micropolar Elasticity," Indian Univ. Math. J., 15(6), pp. 909-923.

[6] Eringen, A. C., 1990, "Theory of Thermo-Microstretch Elastic Solids," Int. J. Eng. Sci., 28(12), pp. 1291-1301.

[7] Parfitt, V. R., and Eringen, A. C., 1969, "Reflection of Plane Waves From the Flat Boundary of a Micropolar Elastic Half-Space," J. Acoust. Soc. Am., 45(5), pp. $1258-1272$.

[8] Ariman, T., 1972, "Wave Propagation in a Micropolar Elastic Half-Space," Acta Mech., 13(1-2), pp. 11-20.

[9] Tomar, S. K., and Gogna, M. L., 1992, "Reflection and Refraction of a Longitudinal Microrotational Wave at an Interface Between Two Micropolar Elastic Solids in Welded Contact," Int. J. Eng. Sci., 30(11), pp. 1637-1646.

[10] Tomar, S. K., and Gogna, M. L., 1995, "Reflection and Refraction of Coupled Transverse and Micro-Rotational Waves at an Interface Between Two Different Micropolar Elastic Media in Welded Contact," Int. J. Eng. Sci., 33(4), pp. 485-496.
[11] Tomar, S. K., and Kumar, R., 1999, "Wave Propagation at Liquid/Micropolar Elastic Solid Interface," J. Sound Vib., 222(5), pp. 858-869.

[12] Singh, B., 2000, "Reflection of Plane Sound Wave From a Micropolar Generalized Thermoelastic Solid Half-Space," J. Sound Vib., 235(4), pp. 685-696.

[13] Singh, B., 2007, "Wave Propagation in an Orthotropic Micropolar Elastic Solid," Int. J. Solids Struct., 44(11), pp. 3638-3645.

[14] Khurana, A., and Tomar, S. K., 2007, "Propagation of Plane Elastic Waves at a Plane Interface Between Two Electro-Microelastic Solid Half-Spaces," Int. J. Solids Struct., 44(11), pp. 3773-3795.

[15] Tomar, S. K., and Khurana, A., 2008, "Elastic Waves in an ElectroMicroelastic Solid,” Int. J. Solids Struct., 45(1), pp. 276-302.

[16] Chiriță, S., and Ghiba, I. D., 2012, "Rayleigh Waves in Cosserat Elastic Materials," Int. J. Eng. Sci., 51, pp. 117-127.

[17] Kumari, N., 2014, "Longitudinal Wave Propagation at an Imperfect Boundary of Micropolar Viscoelastic Solid and Fluid Saturated Incompressible Porous Solid," Int. J. Stat. Math., 10(3), pp. 73-79.

[18] Zhang, P., Wei, P., and Tang, Q., 2015, "Reflection of Micropolar Elastic Waves at the Non-Free Surface of a Micropolar Elastic Half-Space," Acta Mech., 226(9), pp. 2925-2937.

[19] Hsia, S. Y., and Su, C. C., 2008, "Propagation of Longitudinal Waves in Microporous Slab Sandwiched Between Elastic Half-Spaces,” Jpn. J. Appl. Phys., Part 1, 47(7R), pp. 5581-5590.

[20] Gauthier, R. D., 1982, "Experimental Investigations on Micropolar Media," Mechanics of Micropolar Media, World Scientific, Singapore, pp. 395-463. 